\title{
WEIGHT REPRESENTATIONS OF ADMISSIBLE AFFINE VERTEX ALGEBRAS
}

\author{
TOMOYUKI ARAKAWA, VYACHESLAV FUTORNY, AND LUIS ENRIQUE RAMIREZ
}

\begin{abstract}
For an admissible affine vertex algebra $V_{k}(\mathfrak{g})$ of type $A$, we describe a new family of relaxed highest weight representations of $V_{k}(\mathfrak{g})$. They are simple quotients of representations of the affine Kac-Moody algebra $\widehat{\mathfrak{g}}$ induced from the following $\mathfrak{g}$-modules: 1 ) generic Gelfand-Tsetlin modules in the principal nilpotent orbit, in particular all such modules induced from $\mathfrak{s l}_{2} ; 2$ ) all Gelfand-Tsetlin modules in the principal nilpotent orbit which are induced from $\mathfrak{s l}_{3}$; 3) all simple Gelfand-Tsetlin modules over $\mathfrak{s l}_{3}$. This in particular gives the classification of all simple positive energy weight representations of $V_{k}(\mathfrak{g})$ with finite dimensional weight spaces for $\mathfrak{g}=\mathfrak{s l}_{3}$.
\end{abstract}

\section{INTRODUCTION}

Let $\mathfrak{g}$ be a complex simple finite dimensional Lie algebra, $\widehat{\mathfrak{g}}=\mathfrak{g}\left[t, t^{-1}\right]+\mathbb{C} K$ the non-twisted affine Kac-Moody algebra associated with $\mathfrak{g}, V^{k}(\mathfrak{g})$ the universal affine vertex algebra associated with $\mathfrak{g}$ at a non-critical level $k, V_{k}(\mathfrak{g})$ the unique simple quotient of $V^{k}(\mathfrak{g})$.

The simple affine vertex algebra $V_{k}(\mathfrak{g})$ is called admissible if it is isomorphic to an admissible representation [KW89] as a $\widehat{\mathfrak{g}}$-module. In the case when $\mathfrak{g}=\mathfrak{s l}_{2}$, the modular properties and the Verlinde formula for $V_{k}(\mathfrak{g})$-modules were studied in [Rid09, Rid10, CR12, CR13]. To generalize these results to an arbitrary $\mathfrak{g}$, one needs to classify simple positive energy weight representations of $V_{k}(\mathfrak{g})$, that is, $V_{k}(\mathfrak{g})$-modules that appear as simple quotients of $\widehat{\mathfrak{g}}$-modules induced from simple weight representations of $\mathfrak{g}$ which are not necessarily highest weight.

By a well-known result of Zhu [Zhu96], there is a one-to-one correspondence between simple positive energy representations of a graded vertex algebra $V$ and simple $A(V)$-modules, where $A(V)$ is Zhu's algebra of $V$. In the case when $V$ is an affine vertex algebra $V_{k}(\mathfrak{g})$, we have

$$
A\left(V_{k}(\mathfrak{g})\right) \cong U(\mathfrak{g}) / I_{k}
$$

for some two sided-ideal $I_{k}$ of the universal enveloping algebra $U(\mathfrak{g})$ of $\mathfrak{g}$. Thus, a simple $U(\mathfrak{g})$-module $M$ is an $A\left(V_{k}(\mathfrak{g})\right)$-module if and only if the annihilating ideal of $M$ in $U(\mathfrak{g})$ contains $I_{k}$.

In [Ara16] the first named author classified primitive ideals of $U(\mathfrak{g})$ containing $I_{k}$ for an admissible affine vertex algebra $V_{k}(\mathfrak{g})$, proving the Adamovic-Milas conjecture [AM95]. Such primitive ideals are exactly the annihilators of simple highest weight representations $L(\lambda)$ of $\mathfrak{g}$ that appear as the top weight part of a level $k$ admissible modules $\widehat{L}_{k}(\lambda)$ of $\widehat{\mathfrak{g}}$ that are $V_{k}(\mathfrak{g})$-modules. Hence our problem amounts to classifying simple weight modules of $U(\mathfrak{g})$ having those annihilating ideals. 
This is a difficult problem. Complete classification of simple weight modules of $\mathfrak{g}$ is only known for $\mathfrak{g}=\mathfrak{s l}_{2}$. It remains open for all other simple Lie algebras although certain categories of modules were studied extensively (finite dimensional modules, category $\mathcal{O}$ etc.) In particular, simple weight modules which have finite dimensional weight spaces have been classified by Fernando [Fe90] and Mathieu [Mat00]. Among weight modules with infinite dimensional weight spaces the most studied is the class of Gelfand-Tsetlin modules for type $A$. On such modules the Gelfand-Tsetlin subalgebra (being a maximal commutative subalgebra) of the universal enveloping algebra of $\mathfrak{s l}_{n}$ has a generalized eigenspace decomposition. Gelfand-Tsetlin modules were introduced in [DFO89], [DFO92], [DFO94]. Gelfand-Tsetlin subalgebras are related to important problems in representation theory of Lie algebras [FO10], [FO14], general hypergeometric functions on the complex Lie group $G L(n)$, [GR04]; problems in classical mechanics, [KW06], [KW6] among the others.

In this paper we describe several new families of simple positive energy weight representations of admissible affine vertex algebra $V_{k}(\mathfrak{g})$ for $\mathfrak{g}=\mathfrak{s l}_{n}$. More precisely, we construct explicitly the following classes of simple weight $A\left(V_{k}(\mathfrak{g})\right)$-modules for $\mathfrak{g}=\mathfrak{s l}_{n}:$

- generic Gelfand-Tsetlin $\mathfrak{s l}_{n}$-modules in the principal nilpotent orbit (Corollary 4.3, Theorem 6.5). Here we say a simple $\mathfrak{g}$-module is in a nilpotent orbit $\mathbb{O}$ if the associated variety of its annihilating ideal is the closure of $\mathbb{O}$. In this class of representations those with finite dimensional weight spaces are either highest weight modules or induced from an $\mathfrak{s l}_{2}$-subalgebra. Otherwise they have infinitedimensional weight spaces;

- $\mathfrak{s l}_{n}$-modules induced from simple Gelfand-Tsetlin $\mathfrak{s l}_{3}$-modules in the principal nilpotent orbit (Theorem 6.7). All these module have infinite dimensional weight spaces;

- Gelfand-Tsetlin $\mathfrak{s l}_{3}$-modules (Theorem 5.6 and Theorem 5.8). We give a complete classification of Gelfand-Tsetlin $A\left(V_{k}(\mathfrak{g})\right)$-module for $\mathfrak{g}=\mathfrak{s l}_{3}$. This includes all simple weight modules with finite weight multiplicities (both cuspidal and induced) as well as series of modules with infinite weight multiplicities.

Hence we obtain in particular a complete classification of all simple relaxed highest weight representations with finite dimensional weight spaces of admissible affine vertex algebras $V_{k}(\mathfrak{g})$ for $\mathfrak{g}=\mathfrak{s l}_{3}$. We note that a family of weight modules for $V_{k}\left(\mathfrak{s l}_{3}\right)$ has been previously constructed in [Ad16].

Our approach is based on the theory of Gelfand-Tsetlin modules developed in [FGR], [FGR15], [FGR16]. In these papers explicit basis was constructed for different classes of simple Gelfand-Tsetlin modules for $\mathfrak{g l}_{n}$, including generic and 1singular cases. This basis generalizes the classical Gelfand-Tsetlin basis for finite dimensional representations. In [FGR] a complete classification and explicit construction of all simple Gelfand-Tsetlin modules for $\mathfrak{s l}_{3}$ is given. In all other cases the problem of classifying the simple Gelfand-Tsetlin modules is still open.

Acknowledgments. The authors thank the anonymous referees for careful reading and helpfull comments. T.A. is supported in part by the Fapesp grant (2015/06469-6) and by JSPS KAKENHI Grant Numbers 25287004 and 26610006. He gratefully acknowledges the hospitality and excellent working conditions at the 
São Paulo University where this work was done. V.F. is supported in part by the CNPq grant (301320/2013-6) and by the Fapesp grant (2014/09310-5).

\section{Weight MOdUles AND ADMISSIBle REPRESENTATIONS}

2.1. Weight modules. Let $\mathfrak{g}$ be a complex simple finite dimensional Lie algebra with a fixed triangular decomposition $\mathfrak{g}=\mathfrak{n}_{-} \oplus \mathfrak{h} \oplus \mathfrak{n}_{+}$. A $\mathfrak{g}$-module $M$ is called weight if $\mathfrak{h}$ is diagonalizable on $M$. For $\lambda \in \mathfrak{h}^{*}$ the subspace $M_{\lambda}$ of those $v \in V$ such that $h v=\lambda(h) v$ is the weight subspace of weight $\lambda$. The set of all those $\lambda \in \mathfrak{h}^{*}$ for which $M_{\lambda} \neq 0$ is the weight support of $M$ and the dimension of $M_{\lambda}$ is the weight multiplicity of $\lambda$.

Let $\mathfrak{p}=\mathfrak{l} \oplus \mathfrak{m}$ be a parabolic subalgebra of $\mathfrak{g}$ with the Levi subalgebra ll. If $N$ is a weight module over $[\mathfrak{l}, \mathfrak{l}]$ then one defines on it a structure of a $\mathfrak{l}$-module by choosing any $\lambda \in\left(\mathfrak{h}^{\perp}\right)^{*}$ and setting $h v=\lambda(h) v$ for any $h \in \mathfrak{h}^{\perp}$ and any $v \in N$. Here, $\mathfrak{h}^{\perp}$ is the orthogonal complement of $[\mathfrak{l}, \mathfrak{l}] \cap \mathfrak{h}$ with respect to the Killing form. Furthermore, consider $N$ as a $\mathfrak{p}$-module with a trivial action of $\mathfrak{m}$.

Now construct the induced $\mathfrak{g}$-module $M_{\mathfrak{p}}(\lambda, N)=U(\mathfrak{g}) \otimes_{U(\mathfrak{p})} N$. If $N$ is simple $[\mathfrak{l}, \mathfrak{l}]$-module then $M_{\mathfrak{p}}(\lambda, N)$ has a unique simple quotient $L_{\mathfrak{p}}(\lambda, N)$.

When $\mathfrak{p}$ is the fixed Borel subalgebra $\mathfrak{h}+\mathfrak{n}_{+}$, we write $M(\lambda)$ for $M_{\mathfrak{p}}(\lambda, \mathbb{C})$ and $L(\lambda)$ for $L_{\mathfrak{p}}(\lambda, \mathbb{C})$.

We say that a simple weight $\mathfrak{g}$-module $L$ is cuspidal if it is not isomorphic to $L_{\mathfrak{p}}(\lambda, N)$ for any choice of a parabolic subalgebra $\mathfrak{p} \neq \mathfrak{g}$ and any choice of $N$. By [DMP00], if $L$ is cuspidal then its weight support coincides with a coset of some weight (any weight from the support) by the root lattice.

\subsection{Affine vertex algebras. Let}

$$
\widehat{\mathfrak{g}}=\mathfrak{g}\left[t, t^{-1}\right] \oplus \mathbb{C} K
$$

be the affine Kac-Moody algebra associated with $\mathfrak{g}$. The commutation relation of $\widehat{\mathfrak{g}}$ is given by

$$
\left[x t^{m}, y t^{n}\right]=[x, y] t^{m+n}+m(x \mid y) \delta_{m+n, 0} K, \quad[K, \widehat{\mathfrak{g}}]=0,
$$

where $(\mid)$ is the normalized invariant bilinear form of $\mathfrak{g}$.

For $k \in \mathbb{C}$, let $V^{k}(\mathfrak{g})$ be the universal affine vertex algebra associated with $\mathfrak{g}$ at level $k$. By definition

$$
V^{k}(\mathfrak{g})=U(\widehat{\mathfrak{g}}) \otimes_{U(\mathfrak{g}[t] \oplus \mathbb{C} K)} \mathbb{C}_{k},
$$

where $\mathbb{C}_{k}$ is the one-dimensional representation of $\mathfrak{g}[t] \oplus \mathbb{C} K$ on which $\mathfrak{g}[t]$ acts trivially and $K$ acts as a multiplication by $k$. The space $V^{k}(\mathfrak{g})$ is equipped with the natural vertex algebra structure (see [Kac98, FBZ04]), and it is conformal by the Sugawara construction provided that $k \neq-h^{\vee}$, where $h^{\vee}$ is the dual Coxeter number of $\mathfrak{g}$. The unique simple graded quotient $V_{k}(\mathfrak{g})$ of $V^{k}(\mathfrak{g})$ is called the simple affine vertex algebra associated with $\mathfrak{g}$ at level $k$.

For a conformal vertex algebra $V$, let $A(V)$ be Zhu's algebra of $V$. For a positive energy representation $M$ of $V$, the top conformal weight space $M_{t o p}$ is naturally a module over $A(V)$, and moreover, the correspondence $M \mapsto M_{t o p}$ gives a one-toone correspondence between simple positive energy representation of $V$ and simple $V$-modules ([Zhu96]).

In the case that $V=V^{k}(\mathfrak{g})$, we have the natural isomorphism

$$
A\left(V^{k}(\mathfrak{g})\right) \cong U(\mathfrak{g}),
$$


and hence,

$$
A\left(V_{k}(\mathfrak{g})\right) \cong U(\mathfrak{g}) / I_{k}
$$

for some two-sided ideal $I_{k}$ of $U(\mathfrak{g})$.

A $V_{k}(\mathfrak{g})$-module is called weight it is a direct sum of weight modules over $\mathfrak{g}$. In this paper we study the positive energy weight representations of an admissible affine vertex algebra $V_{k}(\mathfrak{g})$.

For a simple weight module $E$ of $\mathfrak{g}$, let $\mathbf{M}_{k}(E)=U(\widehat{\mathfrak{g}}) \otimes_{U(\mathfrak{g}[t] \oplus \mathbb{C K})} E$, where $E$ is considered as a $\mathfrak{g}[t] \oplus \mathbb{C} K$-module on which $\mathfrak{g}[t] t$ acts trivially and $K$ acts the multiplication by $k$. It is clear that $\mathbf{M}_{k}(E)$ is a weight module and so is its simple quotient $\mathbf{L}_{k}(E)$.

According to Zhu's Theorem above the correspondence $E \mapsto \mathbf{L}_{k}(E)$ gives the one to one correspondences between simple weight representations of $A\left(V_{k}(\mathfrak{g})\right)=$ $U(\mathfrak{g}) / I_{k}$ and simple relaxed positive energy highest weight representations of $V_{k}(\mathfrak{g})$.

A positive energy weight representation is an example of a relaxed highest representation [FST] of $\widehat{\mathfrak{g}}$. A general simple relaxed highest representation is obtained from a simple positive energy weight representation by applying a spectral flow.

2.3. Admissible representations of $\widehat{\mathfrak{g}}$. Let $\Delta$ be the set of roots, $\Delta_{+}$the set of positive roots, $W$ the Weyl group of $\mathfrak{g}$. Put $Q=\sum_{\alpha \in \Delta} \mathbb{Z} \alpha, Q^{\vee}=\sum_{\alpha \in \Delta} \mathbb{Z} \alpha^{\vee}$, where $\alpha^{\vee}=2 \alpha /(\alpha, \alpha)$. Let $P$ be the weight lattice of $\mathfrak{g}, P^{\vee}$ the coweight lattice. Denote by $\theta$ and $\theta_{s}$ the highest root and the highest short root of $\mathfrak{g}$, respectively. We have $(\theta, \theta)=2$ and $\left(\theta_{s}, \theta_{s}\right)=2 / r^{\vee}$, where $r^{\vee}$ is the lacing number of $\mathfrak{g}$, that is, the maximum number of the edges in the Dynkin diagram of $\mathfrak{g}$. Let $\rho$ be the half sum of positive roots, $\rho^{\vee}$ the half sum of positive coroots.

We fix the Cartan subalgebra $\widehat{\mathfrak{h}}$ of $\widehat{\mathfrak{g}}$ as $\widehat{\mathfrak{h}}=\mathfrak{h} \oplus \mathbb{C} K$. We will extend $\widehat{\mathfrak{g}}$ by a derivation $D$ and denote the extended algebra by $\widetilde{\mathfrak{g}}: \widetilde{\mathfrak{g}}=\mathfrak{g}\left[t, t^{-1}\right] \oplus \mathbb{C} K+\mathbb{C} D$. Let $\widetilde{\mathfrak{h}}=\mathfrak{h} \oplus \mathbb{C} K \oplus \mathbb{C} D$ be the extended Cartan subalgebra of $\widetilde{\mathfrak{g}}, \widetilde{\mathfrak{h}}^{*}=\mathfrak{h}^{*} \oplus \mathbb{C} \Lambda_{0} \oplus \mathbb{C} \delta$ its dual, where $\Lambda_{0}(K)=\delta(D)=1, \Lambda(\mathfrak{h}+\mathbb{C} D)=\delta(\mathfrak{h} \oplus C K)=0$. The dual $\widehat{\mathfrak{h}}^{*}$ of $\widehat{\mathfrak{h}}$ is identified with the subspace $\mathfrak{h} \oplus \mathbb{C} \Lambda_{0} \subset \widetilde{\mathfrak{h}}^{*}$.

Let $\widehat{\Delta}$ be the set of roots of $\widehat{\mathfrak{g}}$ in $\widetilde{\mathfrak{h}}^{*}, \widehat{\Delta}^{r e} \subset \widehat{\Delta}$ the set of real root of $\widehat{\mathfrak{g}}, \widehat{\Delta}_{+}^{r e}$ the set of real positive roots. We have $\widehat{\Delta}^{r e}=\{\alpha+n \delta \mid \alpha \in \Delta, n \in \mathbb{Z}\}, \widehat{\Delta}_{+}^{r e}=\{\alpha+n \delta \mid$ $\left.\alpha \in \Delta_{+}, n \in \mathbb{Z}_{\geqslant 0}\right\} \sqcup\left\{-\alpha+n \delta \mid \alpha \in \Delta_{+}, n \in \mathbb{Z}_{\geqslant 1}\right\}$.

Denote by $\widehat{W}$ the Weyl group of $\widehat{\mathfrak{g}}$. Then $\widehat{W}=W \ltimes Q^{\vee}$. Let $\widetilde{W}=W \ltimes P^{\vee}$, the extended affine Weyl group of $\mathfrak{g}$. For $\mu \in P^{\vee}$, we denote by $t_{\mu}$ the corresponding element of $\widetilde{W}$. We have $\widetilde{W}=\widetilde{W}_{+} \ltimes \widehat{W}$, where $\widetilde{W}_{+}$is a finite subgroup of $\widetilde{W}$ consisting of elements of length zero. The group $\widetilde{W}_{+}$is described as follows. Write $\theta$ as a sum of simple roots: $\theta=\sum_{i=1}^{l} a_{i} \alpha_{i}$, and set $J=\left\{i \in\{1, \ldots, l\} ; a_{i}=1\right\}$. Then we have

$$
\widetilde{W}_{+}=\left\{t_{\varpi_{j}} w_{j} ; j \in J\right\},
$$

where $\varpi_{j}$ is the $j$-th fundamental weight of $\mathfrak{g}$ and $w_{j}$ is the unique element of $W$ which fixes the set $\left\{\alpha_{1}, \ldots, \alpha_{l},-\theta\right\}$ and $w_{j}(-\theta)=\alpha_{j}$.

For $\lambda \in \widehat{\mathfrak{h}}^{*}$, let $\widehat{\Delta}(\lambda)$ and $\widehat{W}(\lambda)$ be its integral root system and its integral Weyl group, respectively, that is,

$$
\widehat{\Delta}(\lambda)=\left\{\alpha \in \widehat{\Delta}^{r e} ;\left\langle\lambda+\widehat{\rho}, \alpha^{\vee}\right\rangle \in \mathbb{Z}\right\}, \quad \widehat{W}(\lambda)=\left\langle s_{\alpha} ; \alpha \in \widehat{\Delta}(\lambda)\right\rangle \subset \widehat{W},
$$


where $s_{\alpha}$ is the reflection corresponding to $\alpha$ and $\widehat{\rho}=\rho+h^{\vee} \Lambda_{0}$. Let $\widehat{\Delta}(\lambda)_{+}=$ $\widehat{\Delta}(\lambda) \cap \widehat{\Delta}_{+}^{r e}$, the set of positive roots of $\widehat{\Delta}(\lambda)$ and $\widehat{\Pi}(\lambda) \subset \widehat{\Delta}(\lambda)_{+}$, the set of simple roots.

For $\lambda \in \mathfrak{h}^{*}$ and $k \in \mathbb{C}$, set $\widehat{L}_{k}(\lambda):=\mathbf{L}_{k}(L(\lambda))$, which is the irreducible highest weight representation of $\widehat{\mathfrak{g}}$ with highest weight $\widehat{\lambda}:=\lambda+k \Lambda_{0} \in \widehat{\mathfrak{h}}^{*}$.

Recall that a weight $\lambda \in \widehat{\mathfrak{h}}^{*}$ is called admissible [KW89] if

(1) $\lambda$ is regular dominant, that is, $\left\langle\lambda+\widehat{\rho}, \alpha^{\vee}\right\rangle \notin\{0,-1,-2, \ldots\}$ for all $\alpha \in \widehat{\Delta}_{+}^{r e}$;

(2) $\mathbb{Q} \widehat{\Delta}(\lambda)=\mathbb{Q} \widehat{\Delta}^{r e}$.

The simple highest weight representation $\widehat{L}_{k}(\lambda)$ called admissible if $\widehat{\lambda}$ is admissible.

A complex number $k$ is called admissible (for $\widehat{\mathfrak{g}}$ ) if the affine vertex algebra $V_{k}(\mathfrak{g})$ is admissible as an $\widehat{\mathfrak{g}}$-module. (Note that $V_{k}(\mathfrak{g}) \cong \widehat{L}_{k}(0)$ ).

Proposition 2.1 ([KW89, KW08]). The number $k$ is admissible if and only if

$$
k+h^{\vee}=\frac{p}{q} \quad \text { with } p, q \in \mathbb{N},(p, q)=1, p \geqslant \begin{cases}h^{\vee} & \text { if }\left(r^{\vee}, q\right)=1 \\ h & \text { if }\left(r^{\vee}, q\right)=r^{\vee},\end{cases}
$$

where $h$ is the Coxeter number of $\mathfrak{g}$. If this is the case we have $\widehat{\Pi}\left(k \Lambda_{0}\right)=\left\{\dot{\alpha_{0}}, \alpha_{1}, \alpha_{2}, \ldots, \alpha_{l}\right\}$, where

$$
\dot{\alpha_{0}}= \begin{cases}-\theta+q \delta & \text { if }\left(r^{\vee}, q\right)=1 \\ -\theta_{s}+\frac{q}{r^{\vee}} \delta & \text { if }\left(r^{\vee}, q\right)=r^{\vee} .\end{cases}
$$

For an admissible number $k$, let $P r_{k}$ be the set of admissible weights $\lambda$ such that there exists $y \in \widetilde{W}$ satisfying $\widehat{\Delta}(\lambda)=y\left(\widehat{\Delta}\left(k \Lambda_{0}\right)\right)$. Set

$$
\operatorname{Pr}_{k, \mathbb{Z}}=\operatorname{Pr}_{k} \cap \widehat{P}_{k},
$$

where

$$
\widehat{P}_{k}=\left\{\lambda \in \widehat{\mathfrak{h}}^{*} ; \lambda(K)=k,\left\langle\lambda, \alpha_{i}^{\vee}\right\rangle \in \mathbb{Z} \text { for all } i=1, \ldots, l\right\}
$$

Then

$$
\operatorname{Pr}_{k, \mathbb{Z}}= \begin{cases}\left\{\lambda \in \widehat{P}_{k} ;\left\langle\lambda, \alpha_{i}^{\vee}\right\rangle \geqslant 0 \text { for } i=1, \ldots, l,\langle\lambda, \theta\rangle \leqslant p-h^{\vee}\right\} & \text { if }\left(r^{\vee}, q\right)=1, \\ \left\{\lambda \in \widehat{P}_{k} ;\left\langle\lambda, \alpha_{i}^{\vee}\right\rangle \geqslant 0 \text { for } i=1, \ldots, l,\left\langle\lambda, \theta_{s}^{\vee}\right\rangle \leqslant p-h\right\} & \text { if }\left(r^{\vee}, q\right)=r^{\vee} .\end{cases}
$$

Note that

$$
\operatorname{Pr}_{k, \mathbb{Z}} \cong \begin{cases}\widehat{P}_{+}^{p-h^{\vee}} & \text { if }\left(r^{\vee}, q\right)=1, \\ { }^{L} \widehat{P}_{+}^{\vee, p-h} & \text { if }\left(r^{\vee}, q\right)=r^{\vee},\end{cases}
$$

where $\widehat{P}_{+}^{m}$ is the set of level $m$ integral dominant weights of $\widehat{\mathfrak{g}}$, and ${ }^{L} \widehat{P}_{+}^{\vee, m}$ is the set of level $m$ integral dominant coweights of the affine Kac-Moody algebra $\widehat{L_{\mathfrak{g}}}$ associated with the Langlands dual Lie algebra ${ }^{L} \mathfrak{g}$.

For $\lambda \in P r_{k, \mathbb{Z}}$ we have

$$
\widehat{\Delta}(\lambda)= \begin{cases}\{\alpha+n q \delta ; \alpha \in \Delta, n \in \mathbb{Z}\} & \text { if }\left(q, r^{\vee}\right)=1, \\ \left\{\alpha+n q \delta ; \alpha \in \Delta_{\text {long }}\right\} \sqcup\left\{\alpha+\frac{n q}{r \vee} \delta ; \alpha \in \Delta_{\text {short }}, n \in \mathbb{Z}\right\} & \text { if }\left(q, r^{\vee}\right)=r^{\vee},\end{cases}
$$


where $\Delta_{\text {long }}$ (resp. $\Delta_{\text {short }}$ ) is the set of long roots (resp. short roots) of $\mathfrak{g}$. It follows that

$$
\widehat{W}(\lambda)= \begin{cases}W \ltimes q Q^{\vee} & \text { if }\left(q, r^{\vee}\right)=1, \\ W \ltimes q Q & \text { if }\left(q, r^{\vee}\right)=r^{\vee}\end{cases}
$$

for $\lambda \in P r_{k, \mathbb{Z}}$. In particular

$$
\widehat{W}(\lambda) \cong \begin{cases}\widehat{W} & \text { if }\left(q, r^{\vee}\right)=1, \\ { }^{L} \widehat{W} & \text { if }\left(q, r^{\vee}\right)=r^{\vee},\end{cases}
$$

for $\lambda \in P r_{k}$, where ${ }^{L} \widehat{W}$ is the Weyl group of $\widehat{L_{\mathfrak{g}}}$.

If $k$ is an admissible number with denominator $q$ such that $\left(q, r^{\vee}\right)=1$, we have [KW89]

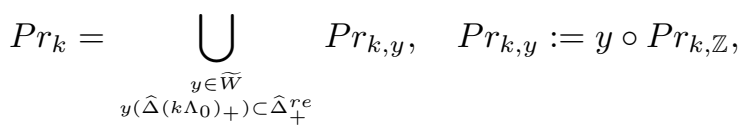

Moreover,

$$
\operatorname{Pr}_{k, y} \cap P r_{k, y^{\prime}} \neq \emptyset \Longleftrightarrow P r_{k, y}=P r_{k, y^{\prime}} \Longleftrightarrow y^{\prime}=y t_{q \varpi_{j}} w_{j}
$$

with some $j \in J$ for $y, y^{\prime} \in \widetilde{W}$ such that $y\left(\widehat{\Delta}\left(k \Lambda_{0}\right)_{+}\right), y^{\prime}\left(\widehat{\Delta}\left(k \Lambda_{0}\right)_{+}\right) \subset \widehat{\Delta}_{+}^{r e}$.

2.4. Admissible representations and nilpotent orbits. Let $k$ be an admissible number for $\widehat{\mathfrak{g}}$.

We extend the concept of admissibility to modules over $\mathfrak{g}$. Namely, we say that a $\mathfrak{g}$-module $M$ is admissible of level $k$ if $\mathbf{L}(M)$ is an $V_{k}(\mathfrak{g})$-module, or equivalently, $M$ is an $A\left(V_{k}(\mathfrak{g})\right)$-module. Thus, $M \mapsto \mathbf{L}(M)$ gives a one-to-one correspondence between the set of the isomorphism classes of admissible $\mathfrak{g}$-modules of level $k$ and that of simple positive energy representations of $V_{k}(\mathfrak{g})$.

Let

$$
\overline{P r_{k}}=\left\{\bar{\lambda} \mid \lambda \in P r_{k}\right\} \subset \mathfrak{h}^{*},
$$

where $\bar{\lambda}$ is the projection of $\lambda$ to $\mathfrak{h}^{*}$.

Theorem 2.2 ([Ara16]). Let $k$ be admissible, $\lambda \in \mathfrak{h}^{*}$. Then $\widehat{L}_{k}(\lambda)$ is a module over $V_{k}(\mathfrak{g})$ if and only if $\lambda \in \overline{P r_{k}}$. Equivalently, $L(\lambda)$ is a $A\left(V_{k}(\mathfrak{g})\right)$-module if and only if $\lambda \in \overline{P r_{k}}$.

Theorem 2.2 in particular says that any $A\left(V_{k}(\mathfrak{g})\right)$-module in the category $\mathcal{O}$ is completely reducible since all elements of $\overline{P r_{k}}$ are regular dominant, that is, $\left\langle\lambda+\rho, \alpha^{\vee}\right\rangle \notin\{0,-1,-2,-3, \ldots\}$ for all $\alpha \in \Delta_{+}$.

For $\lambda \in \mathfrak{h}^{*}$, let

$$
J_{\lambda}=\operatorname{Ann}_{U(\mathfrak{g})} L(\lambda) .
$$

By Duflo's Theorem [Duf77], one knows that for any primitive ideal $I$ (that is, the annihilator of some simple module) of $U(\mathfrak{g})$ there exists $\lambda \in \mathfrak{h}^{*}$ such that $I=J_{\lambda}$.

Thus, we obtain following assertion immediately from Theorem 2.2.

Corollary 2.3. Let $k$ be an admissible number, $M$ a simple $U(\mathfrak{g})$-module. Then $M$ is an admissible $\mathfrak{g}$-module of level $k$ if and only if $\operatorname{Ann}_{U(\mathfrak{g})} M=J_{\lambda}$ for some $\lambda \in \overline{P r_{k}}$. 
Let $\mathcal{Z}(\mathfrak{g})$ be the center of $U(\mathfrak{g})$, and let $\chi_{\lambda}: \mathcal{Z}(\mathfrak{g}) \rightarrow \mathbb{C}$ be the evaluation at $L(\lambda)$. By [Jos79], one knows that the correspondence $I \mapsto I M(\lambda)$ gives an order-preserving injection between the set of two sided ideals of $U(\mathfrak{g})$ containing $U(\mathfrak{g})$ ker $\chi_{\lambda}$ and submodules of $M(\lambda)$, and in fact, this map is a bijection if $\lambda$ is regular.

Proposition 2.4. For $\lambda \in \overline{P r_{k}}$, the primitive ideal $J_{\lambda}$ is the unique maximal twosided ideal of $U(\mathfrak{g})$ containing $U(\mathfrak{g}) \operatorname{ker} \chi_{\lambda}$. In particular, for $\lambda, \mu \in \overline{P r_{k}}, J_{\lambda}=J_{\mu}$ if and only if there exists $w \in W$ such that $\mu=w \circ \lambda$.

Proof. Let $\lambda \in \overline{P r_{k}}$. Then $I_{k} \subset J_{\lambda}$ by Theorem 2.2. (Recall that $A\left(L_{k}(\mathfrak{g})\right)=$ $\left.U(\mathfrak{g}) / I_{k}\right)$. Thus, $M(\lambda) / J_{\lambda}$ is a highest weight $A\left(L_{k}(\mathfrak{g})\right)$-module, and therefore, it must be isomorphic to $L(\lambda)$ by Theorem 2.2. This means that $J_{\lambda} M(\lambda)$ is the unique maximal submodule of $M(\lambda)$, and therefore, $J_{\lambda}$ is the unique maximal two-sided ideal of $U(\mathfrak{g})$ containing $U(\mathfrak{g})$ ker $\chi_{\lambda}$.

For a two-sided ideal $I$ of $U(\mathfrak{g})$, let $\operatorname{Var}(I)$ be the associated variety of $I$, that is, the zero locus of $\operatorname{gr} I$ in $\mathfrak{g}^{*}$, where gr $I$ is the associated graded with respect to the filtration induced from the PBW filtration of $U(\mathfrak{g})$. By Joseph's Theorem [Jos85], for a primitive ideal $I$ we have $\operatorname{Var}(I)=\overline{\mathbb{O}}$ for some nilpotent orbit $\mathbb{O}$ of $\mathfrak{g}$.

We say a simple $\mathfrak{g}$-module $M$ is in the orbit $\mathbb{O}$ if $\operatorname{Var}\left(\operatorname{Ann}_{U(\mathfrak{g})} M\right)=\overline{\mathbb{O}}$.

Theorem 2.5 ([Ara15b]). Let $k$ be an admissible number for $\widehat{\mathfrak{g}}$ with denominator $q \in \mathbb{N}$. There exists a nilpotent orbit $\mathbb{O}_{q}$ that depends only on $q$ such that

$$
\operatorname{Var}\left(I_{k}\right)=\overline{\mathbb{O}_{q}} \text {. }
$$

Explicitly, we have

$$
\overline{\mathbb{O}}_{q}= \begin{cases}\left\{x \in \mathfrak{g} ;(\operatorname{ad} x)^{2 q}=0\right\} & \text { if }\left(r^{\vee}, q\right)=1, \\ \left\{x \in \mathfrak{g} ; \pi_{\theta_{s}}(x)^{2 q / r^{\vee}}=0\right\} & \text { if }\left(r^{\vee}, q\right)=r^{\vee},\end{cases}
$$

where $r^{\vee}$ is the lacing number of $\mathfrak{g}, \theta_{s}$ is the highest short root of $\mathfrak{g}$ and $\pi_{\theta_{s}}$ is the simple finite-dimensional representation of $\mathfrak{g}$ with highest weight $\theta_{s}$.

See $\left[\right.$ Ara15a] for a more concrete description of the orbits $\mathbb{O}_{q}$.

Let $q \in \mathbb{N}$ be the denominator $q$ of $k$. For a nilpotent orbit $\mathbb{O}$ of $\mathfrak{g}$, set

$$
\overline{\operatorname{Pr}}_{k}^{\mathbb{D}}=\left\{\lambda \in \overline{\operatorname{Pr}}_{k} \mid \operatorname{Var}\left(J_{\lambda}\right)=\overline{\mathbb{O}}\right\}
$$

For $\lambda \in \overline{\operatorname{Pr}}_{k}$ we have $\operatorname{Var}\left(J_{\lambda}\right) \subset \operatorname{Var}\left(I_{k}\right)=\overline{\mathbb{O}_{q}}$ since $I_{k} \subset J_{\lambda}$. Hence

$$
\overline{\operatorname{Pr}}_{k}=\bigsqcup_{\mathbb{O} \subset \overline{\mathbb{O}_{q}}} \overline{\operatorname{Pr}}_{k}^{\mathbb{O}} \text {. }
$$

Note that

$$
\overline{\operatorname{Pr}}_{k}^{\{0\}}=\overline{\operatorname{Pr}}_{k, \mathbb{Z}}:=\left\{\bar{\lambda} \mid \lambda \in \operatorname{Pr}_{k, \mathbb{Z}}\right\} .
$$

We define an equivalence relation in $\overline{P r}_{k}$ by

$$
\lambda \sim \mu \Longleftrightarrow \text { there exists } w \in W \text { such that } \mu=w \circ \lambda \text {. }
$$

$$
\left[\overline{\operatorname{Pr}}_{k}\right]=\overline{\operatorname{Pr}}_{k} / \sim
$$


By Proposition 2.4, $J_{\lambda}$ depends only on the class of $\lambda \in \overline{\operatorname{Pr}}_{k}$ in $\left[\overline{\operatorname{Pr}}_{k}\right]$. We have

$$
\left[\overline{\operatorname{Pr}}_{k}\right]=\bigsqcup_{\mathbb{O} \subset \overline{\mathbb{O}}_{q}}\left[\overline{\operatorname{Pr}}_{k}^{\mathbb{O}}\right]
$$

where $\left[\overline{\operatorname{Pr}}_{k}^{\circledR}\right]$ is the image of $\overrightarrow{\operatorname{Pr}}_{k}^{\circledR}$ in $\left[\overline{\operatorname{Pr}}^{k}\right]$.

We conclude that a simple $U(\mathfrak{g})$-module $M$ in the orbit $\mathbb{O}$ is an admissible $\mathfrak{g}$ module of level $k$ if and only if $\operatorname{Ann}_{U(\mathfrak{g})} M=J_{\lambda}$ for some $\lambda \in\left[\overline{\operatorname{Pr}}_{k}^{\mathbb{Q}}\right]$.

2.5. Principal nilpotent orbit. Let $k$ be an admissible number with denominator $q \in \mathbb{N}$, and let $\mathbb{O}_{\text {prin }}$ be the principal nilpotent orbit, that is, the unique dense orbit in the nilpotent cone $\mathcal{N}$ of $\mathfrak{g}^{*}$. We have $\operatorname{Var}\left(J_{\lambda}\right)=\overline{\mathbb{O}_{\text {prin }}}=\mathcal{N}$ if and only if

$$
\operatorname{Ann}_{U(\mathfrak{g})} L(\lambda)=U(\mathfrak{g}) \operatorname{ker} \chi_{\lambda} .
$$

Let $\lambda \in \overline{\operatorname{Pr}}_{k}$. Since admissible weights are regular dominant, $\lambda \in \overline{\operatorname{Pr}}_{k}{ }_{\text {prin }}$ if and only if $\widehat{\lambda}=\lambda+k \Lambda_{0}$ is non-degenerate in the sense of [FKW92], that is, $\widehat{\Delta}(\lambda) \cap \Delta=\emptyset$, so that $L(\lambda)=M(\lambda)$.

Clearly, the set $\overline{P r}_{k} \mathbb{O}_{\text {prin }}$ is non-empty if and only if $\mathbb{O}_{q}=\mathbb{O}_{\text {prin }}$. By Theorem 2.5 this holds if and only if

$$
q \geqslant \begin{cases}h & \text { if }\left(r^{\vee}, q\right)=1, \\ r^{\vee} h^{\vee} & \text { if }\left(r^{\vee}, q\right)=r^{\vee},\end{cases}
$$

where $h$ is the Coxeter number of $\mathfrak{g}$ and ${ }^{L} h^{\vee}$ is the dual Coxeter number of the Langlands dual ${ }^{L} \mathfrak{g}$ of $\mathfrak{g}$.

Proposition 2.6. ([FKW92]) Let $k$ be an admissible number for $\widehat{\mathfrak{g}}, q \in \mathbb{N}$ the denominator of $k$. Suppose that $\left(r^{\vee}, q\right)=1$ and $q \geqslant h$. We have a bijection

$$
\begin{array}{cccc}
\left(\widehat{P}_{+}^{p-h^{\vee}} \times \widehat{P}_{+}^{\vee, q-h}\right) / \widetilde{W}_{+} & \stackrel{\sim}{\rightarrow} & {\left[\widetilde{P r}_{k}^{\mathbb{P}_{\text {prin }}}\right],} \\
{[(\lambda, \mu)]} & \mapsto & {\left[\bar{\lambda}-\frac{p}{q}\left(\bar{\mu}+\rho^{\vee}\right)\right],}
\end{array}
$$

where $\widetilde{W}_{+}$acts diagonally on $\widehat{P}_{+}^{p-h^{\vee}} \times{ }^{L} \widehat{P}_{+}^{q-h}$.

There is a similar description for the cases that $\left(q, r^{\vee}\right)=r^{\vee}$, but we omit it since we will not need it in this paper.

2.6. Minimal nilpotent orbit in type $A$. Let $\mathfrak{g}=\mathfrak{s l}_{n}$.

Lemma 2.7. Let $\bar{y} \in W$. If $\bar{y} \neq 1$, then there exists $j \in J$ such that $\bar{y} \bar{\pi}_{j}(\theta) \in \Delta_{+}$.

Proof. We have $\bar{y} \bar{\pi}_{j}(\theta)=-\bar{y}\left(\alpha_{j}\right)$. Since $J=\{1,2, \ldots, n-1\}$ for $\mathfrak{g}=\mathfrak{s l}_{n}$ and $\bar{y} \neq 1$, there exists $j \in J$ such that $\bar{y}\left(\alpha_{j}\right) \in \Delta_{-}$.

Recall the decomposition (16). Set $\overline{P r}_{k, y}=\left\{\bar{\lambda} \mid \lambda \in P r_{k, y}\right\}$ and denote by $\left[\overline{P r}_{k, y}\right]$ the image of $\overline{P r}_{k, y}$ in $\left[\overline{P r}_{k}\right]$.

Proposition 2.8. Let $k$ be an admissible number for $\widehat{\mathfrak{g}}, q \in \mathbb{N}$ the denominator of $k$. We have

$$
\left[\overline{\operatorname{Pr}}_{k}\right]=\bigcup_{\substack{\eta \in P_{+} \\ \theta(\eta) \leqslant q-1}}\left[\overline{\operatorname{Pr}}_{k, t_{-\eta}}\right],
$$

where $P_{+}^{\vee}$ is the set of dominate coweights. 
Proof. Let $y=\bar{y} t_{-\eta} \in \widetilde{W}$ with $y \in W, \eta \in P^{\vee}$. It is straightforward to see that the condition $y\left(\widehat{\Delta}\left(k \Lambda_{0}\right)\right) \subset \widehat{\Delta}_{+}^{r e}$ is equivalent to that

$$
\begin{cases}0 \leqslant \alpha(\eta) \leqslant q-1 & \text { if } \bar{y}(\alpha) \in \Delta_{+}, \\ 1 \leqslant \alpha(\eta) \leqslant q & \text { if } \bar{y}(\alpha) \in \Delta_{-},\end{cases}
$$

for all $\alpha \in \Delta_{+}$.

In particular, for $\eta \in P^{\vee}, t_{-\eta}\left(\widehat{\Delta}\left(k \Lambda_{0}\right)\right) \subset \widehat{\Delta}_{+}^{r e}$ if and only if $\eta \in P_{+}^{\vee}$ and $\theta(\eta) \leqslant$ $q-1$. This together with (16) shows the inclusion $\supset$. Conversely, suppose that $y=\bar{y} t_{-\eta} \in \widetilde{W}$ with $y \in W, \eta \in P^{\vee}$ satisfies that $y\left(\widehat{\Delta}\left(k \Lambda_{0}\right)\right) \subset \widehat{\Delta}_{+}^{r e}$. We claim that we may assume that $\bar{y}(\theta) \in \Delta_{+}$. Indeed, $P r_{k, y}=P r_{k, y t_{q \varpi_{j}}} \bar{\pi}_{j}$ by (17) for $j \in J$, and if $\bar{y}(\theta) \in \Delta_{-}$, then there exists $j \in J$ such that $\bar{y} \bar{\pi}_{j}(\theta) \in \Delta_{+}$by Lemma 2.7. We obtain $\eta \in P_{+}^{\vee}$ and $\theta(\eta) \leqslant q-1$, which completes the proof by Proposition 2.4.

Proposition 2.9. Let $\lambda \in \overline{\operatorname{Pr}}_{k, t_{-\eta}}$ with $\eta \in P_{+}^{\vee}, \theta(\eta) \leqslant q-1$. Then

$$
\operatorname{dim} \operatorname{Var}\left(J_{\lambda}\right)=|\Delta|-|\Delta(\lambda)|,
$$

where $\Delta(\lambda)=\left\{\alpha \in \Delta \mid \lambda\left(\alpha^{\vee}\right) \in \mathbb{Z}\right\}$.

Proof. We have $\Delta(\lambda)=t_{-\eta}\left(\widehat{\Delta}\left(k \Lambda_{0}\right)\right) \cap \Delta=\{\alpha \in \Delta \mid \alpha(\eta)=0\}$. Since $\eta \in P_{+}^{\vee}$, it follows that $\Delta(\lambda)$ a subroot system of $\Delta$ generated by the simple roots $\alpha_{i}$ such that $\alpha_{i}(\eta)=0$. Let $\mathfrak{p}$ be the corresponding parabolic subalgebra of $\mathfrak{g}$ containing $\mathfrak{b}_{+}=\mathfrak{h} \oplus \mathfrak{n}, \mathfrak{m}$ its nilradical, $\mathfrak{l}$ its Levi subalgebra. Denote by $L_{\mathfrak{l}}(\lambda)$ the simple finite-dimensional module of $\mathfrak{l}$ with highest weight $\lambda$. Then [Jan77]

$$
L(\lambda)=U(\mathfrak{g}) \otimes_{U(\mathfrak{p})} L_{\mathfrak{l}}(\lambda)
$$

where $L_{\mathfrak{l}}(\lambda)$ is regarded as a $\mathfrak{p}$-module by the projection $\mathfrak{p} \rightarrow \mathfrak{l}$. It follows that the Gelfand-Kirillov dimension $\operatorname{Dim} L(\lambda)$ of $L(\lambda)$ equals to $\operatorname{dim} \mathfrak{m}=1 / 2(|\Delta|-$ $|\Delta(\lambda)|)$. As $L(\lambda)$ is holonomic, we have $\operatorname{dim} \operatorname{Var}\left(J_{\lambda}\right)=2 \operatorname{Dim} L(\bar{\lambda})=|\Delta|-|\Delta(\lambda)|$ as required.

Let $\mathbb{O}_{\min }$ be the unique minimal non-trivial nilpotent orbit of $\mathfrak{g}$, which is of dimension $2 h^{\vee}-2=2(n-1)$ [Wan99]. We have $\mathbb{O}_{\text {min }}=G . e_{\alpha}$, where $e_{\alpha}$ is a root vector of some root $\alpha$.

Proposition 2.10. Let $\mathfrak{g}=\mathfrak{s l}_{n}, k$ an admissible number with denominator $q$. Then

$$
\left[\overline{\operatorname{Pr}}_{k}^{\mathbb{O}_{\text {min }}}\right]=\bigsqcup_{a=1}^{q-1}\left[\overline{\operatorname{Pr}}_{k, t_{-a \varpi_{1}}}\right]=\bigsqcup_{a=1}^{q-1}\left\{\left[\bar{\lambda}-\frac{a p}{q} \varpi_{1}\right] \mid \lambda \in \widehat{P}_{+}^{p-n}\right\}
$$

Proof. Let $\lambda \in \overline{\operatorname{Pr}}_{t_{-\eta}}^{k}$ with $\eta \in P_{+}^{\vee}, \theta(\eta) \leqslant q-1$. By Proposition 2.9, $\lambda \in \overline{\operatorname{Pr}}_{k}^{\mathbb{O}_{\text {min }}}$ if and only if $|\Delta(\lambda)|=n(n-1)-2(n-1)=(n-1)(n-2)$. This happens if and only if $\Delta(\lambda)$ is a root system of type $A_{n-2}$, or equivalently, $\eta=-a \varpi_{1}$ or $\eta=-a \varpi_{n-1}$ for some $a=1, \ldots, q-1$. Since $\left[\overline{\operatorname{Pr}}_{k, t_{-a \varpi_{1}}}\right]=\left[\overline{\operatorname{Pr}}_{k, t_{-a \varpi_{n-1}}}\right]$ by (17) and Proposition 2.4 , we get that

$$
\left[\overline{\operatorname{Pr}}_{k}^{\mathbb{O}_{\text {min }}}\right]=\bigcup_{a=1}^{q-1}\left[\overline{\operatorname{Pr}}_{k, t_{-a \varpi_{1}}}\right] .
$$

It is straightforward to see that this is a disjoint union.

Note that if $M$ is a cuspidal admissible $\mathfrak{g}$-module of level $k$ with finite dimensional weight spaces then $\operatorname{Ann}_{U(\mathfrak{g})}(M)=J_{\lambda}$ for some $\lambda \in\left[\overline{P r}_{k}^{\mathbb{O}_{\text {min }}}\right]$ ([Mat00]). 
2.7. The $\mathfrak{s l}_{3}$-cases. Let $\mathfrak{g}=\mathfrak{s l}_{3}$. Then the level $k$ is admissible if and only if $k+3=p / q$ with $p, q \in \mathbb{N},(p, q)=1, p \geqslant 3$. We have

$$
\mathbb{O}_{q}= \begin{cases}\mathbb{O}_{\text {prin }} & \text { if } q \geqslant 3, \\ \mathbb{O}_{\text {min }} & \text { if } q=2, \\ 0 & \text { if } q=1\end{cases}
$$

Note that $\operatorname{dim} \mathbb{O}_{\text {prin }}=6, \operatorname{dim} \mathbb{O}_{\text {min }}=4$.

The following assertion follows immediately from Proposition 2.6 and Proposition 2.10 .

Proposition 2.11. Let $k$ be an admissible number for $\widehat{\mathfrak{g}}$, so that $k+3=p / q$, $p, q \in \mathbb{Z}, p \geqslant 3,(p, q)=1$. We have

$$
\begin{aligned}
& {\left[\overline{\operatorname{Pr}}_{k}^{\mathbb{O}_{\text {prin }}}\right]=\left\{\left[\bar{\lambda}-\frac{p}{q}(\bar{\mu}+\rho)\right] \mid \lambda \in \widehat{P}_{+}^{p-3}, \mu \in \widehat{P}_{+}^{q-3}\right\},} \\
& {\left[\overline{\operatorname{Pr}}_{k}^{\mathbb{O}_{\text {min }}}\right]=\left\{\left[\bar{\lambda}-\frac{a p}{q} \varpi_{1}\right] \mid \lambda \in \widehat{P}_{+}^{p-3}, a=1, \ldots, q-1\right\},} \\
& {\left[\overline{\operatorname{Pr}}_{k}^{\{0\}}\right]=\left\{[\bar{\lambda}] \mid \lambda \in \widehat{P}_{+}^{p-3}\right\} .}
\end{aligned}
$$

2.8. Compatibility of admissible $\mathfrak{g}$-modules with the restriction. Let $\mathfrak{p}=$ $\mathfrak{l}+\mathfrak{m}$ be a parabolic subalgebra of $\mathfrak{g}$, where $\mathfrak{l}$ is its Levi subalgebra and $\mathfrak{m}$ is its nilradical. Let $[\mathfrak{l}, \mathfrak{l}]=\sum_{i} \mathfrak{l}_{i}$ be a decomposition into the sum of simple Lie subalgebras. Let $\widehat{\mathfrak{l}}_{i}$ be the affine Kac-Moody algebra associated with $\mathfrak{l}_{i}$. For an admissible number $k$ of $\widehat{\mathfrak{g}}$, define the admissible number $k_{i}$ for $\widehat{\mathfrak{l}}_{i}$ by

$$
k_{i}+h_{i}^{\vee}=\frac{2}{\left(\theta_{i}, \theta_{i}\right)}\left(k+h^{\vee}\right)
$$

where $h_{i}^{\vee}$ is the dual Coxeter number of $\mathfrak{l}_{i}, \theta_{i}$ is the highest root of $\mathfrak{l}_{i}$.

Theorem 2.12. Let $M$ be an admissible $\mathfrak{g}$-module of level $k$. Then $\mathfrak{m}$-invariant subspace $M^{\mathfrak{m}}$ is an admissible module of $\mathfrak{l}_{i}$ of level $k_{i}$ for each $i$.

Proof. Since $M$ is an $A\left(V_{k}(\mathfrak{g})\right)$-module, $\mathbf{L}(M)$ is a $V_{k}(\mathfrak{g})$-module. Hence by [Ara14, Theorem 7.5], the semi-infinite cohomology space $H^{\frac{\infty}{2}+n}\left(\mathfrak{m}\left[t, t^{-1}\right], \mathbf{L}(M)\right)$ is a module over the admissible affine vertex algebra $V_{k_{i}}\left(\mathfrak{l}_{i}\right)$ for all $n, i$. Therefore the top weigh space of $H^{\frac{\infty}{2}+n}\left(\mathfrak{m}\left[t, t^{-1}\right], \mathbf{L}(M)\right)$ is a module over $A\left(V_{k_{i}}\left(\mathfrak{l}_{i}\right)\right)$-module, that is to say, an admissible $\mathfrak{l}_{i}$-module of level $k_{i}$. The assertion follows since the top weight space of $H^{\frac{\infty}{2}+n}\left(\mathfrak{m}\left[t, t^{-1}\right], \mathbf{L}(M)\right), n \geqslant 0$, is isomorphic to $H^{n}(\mathfrak{m}, M)$ as an $\mathfrak{l}$-module provided that $H^{n}(\mathfrak{m}, M)$ is nonzero (see the proof of [Ara16, Lemma 4.3]).

\section{Gelfand-Tsetlin modules}

We recall the definition of a Gelfand-Tsetlin module for $\mathfrak{g l}_{n}$. Let $U=U\left(\mathfrak{g l}_{n}\right)$ and $\left\{E_{i j} \mid 1 \leqslant i, j \leqslant n\right\}$ be the standard basis of $\mathfrak{g l}_{n}$ of elementary matrices. For each $m \leqslant n$ let $\mathfrak{g l}_{m}$ be the Lie subalgebra of $\mathfrak{g l}_{n}$ spanned by $\left\{E_{i j} \mid i, j=1, \ldots, m\right\}$. Then we have the following chain

$$
\mathfrak{g l}_{1} \subset \mathfrak{g l}_{2} \subset \ldots \subset \mathfrak{g l}_{n}
$$

which induces the chain $U_{1} \subset U_{2} \subset \ldots \subset U_{n}$ of the universal enveloping algebras $U_{m}=U\left(\mathfrak{g l}_{m}\right), 1 \leqslant m \leqslant n$. Let $Z_{m}$ be the center of $U_{m}$. Then $Z_{m}$ is the polynomial 
algebra in the $m$ variables $\left\{c_{m k} \mid k=1, \ldots, m\right\}$,

$$
c_{m k}=\sum_{\left(i_{1}, \ldots, i_{k}\right) \in\{1, \ldots, m\}^{k}} E_{i_{1} i_{2}} E_{i_{2} i_{3}} \ldots E_{i_{k} i_{1}},
$$

$m=1, \ldots, n$. Following [DFO94], we call the subalgebra of $U$ generated by $\left\{Z_{m} \mid m=1, \ldots, n\right\}$ the (standard) Gelfand-Tsetlin subalgebra of $U$ and denote it by $\Gamma$. In fact, $\Gamma$ is the polynomial algebra in the $\frac{n(n+1)}{2}$ variables $\left\{c_{i j} \mid 1 \leqslant j \leqslant i \leqslant n\right\}$ ([Zh74]).

Definition 3.1. A finitely generated $U$-module $M$ is called a Gelfand-Tsetlin module (with respect to $\Gamma$ ) if $M$ splits into a direct sum of $\Gamma$-modules:

$$
M=\bigoplus_{\mathrm{m} \in \operatorname{Specm} \Gamma} M(\mathrm{~m})
$$

where

$$
M(\mathrm{~m})=\left\{v \in M \mid \mathrm{m}^{k} v=0 \text { for some } k \geqslant 0\right\} .
$$

All those $\mathrm{m} \in \operatorname{Specm} \Gamma$ for which $M(\mathrm{~m}) \neq 0$ form the Gelfand-Tsetlin support of $M$. The dimension of the subspace $M(\mathrm{~m})$ is the Gelfand-Tsetlin multiplicity of $\mathrm{m}$. Since the Gelfand-Tsetlin subalgebra contains the Cartan subalgebra $\mathfrak{h}$ spanned by $\left\{E_{i i} \mid i=1, \ldots, n\right\}$ then any simple Gelfand-Tsetlin module is a weight module. Note that every weight module with finite dimensional weight subspaces is a Gelfand-Tsetlin module. Indeed, any weight space is invariant under the action of the Gelfand-Tsetlin subalgebra (since $\Gamma$ contains all $E_{i i}$ 's) and hence decomposes into its generalized eigen subspaces. In particular, every highest weight module is a Gelfand-Tsetlin module.

\subsection{Generic Gelfand-Tsetlin modules.}

Definition 3.2. For a vector $v$ in $\mathbb{C}^{\frac{n(n+1)}{2}}$, by $T(v)$ we will denote the following array with entries $\left\{v_{i j}: 1 \leqslant j \leqslant i \leqslant n\right\}$

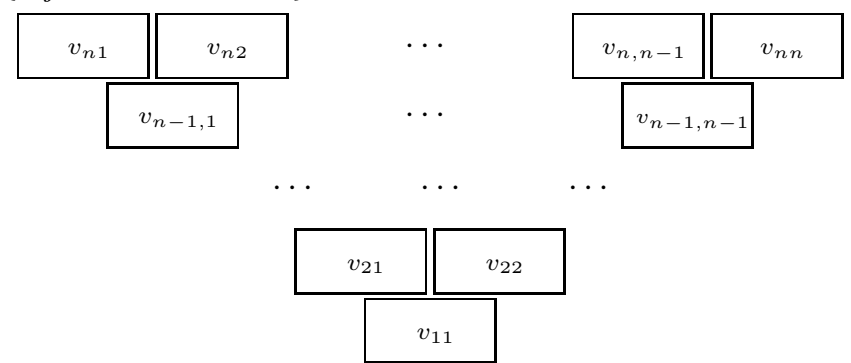

Such an array will be called a Gelfand-Tsetlin tableau of height $n$.

A classical result of Gelfand and Tsetlin [GT50] gives an explicit realization of any simple finite dimensional $\mathfrak{g l}_{n}$-module in terms of Gelfand-Tsetlin tableaux. Using these Gelfand-Tsetlin formulas one can defined the class of infinite dimensional generic modules for $\mathfrak{g l}_{n}$, see for example [DFO94].

Definition 3.3. A vector $v \in \mathbb{C}^{\frac{n(n+1)}{2}}$ and the Gelfand-Tsetlin tableau $T(v)=T\left(v_{i j}\right)$ are called generic if $v_{k i}-v_{k j} \notin \mathbb{Z}$ for all $1 \leqslant i \neq j \leqslant k \leqslant n-1$. It is strongly generic if $v_{r s}-v_{r t} \notin \mathbb{Z}$ for any $1 \leqslant s \neq t \leqslant r \leqslant n$. 
Theorem 3.4 ([DFO94, Section 2.3]). Let $T(v)=T\left(v_{i j}\right)$ be a generic GelfandTsetlin tableau of height $n$. Denote by $\mathcal{B}(T(v))$ the set of all Gelfand-Tsetlin tableaux $T\left(v^{\prime}\right)=T\left(v_{i j}^{\prime}\right)$ satisfying $v_{n j}^{\prime}=v_{n j}, v_{i j}^{\prime}-v_{i j} \in \mathbb{Z}$ for, $1 \leqslant j \leqslant i \leqslant n-1$.

(i) The vector space $V(T(v))=\operatorname{span} \mathcal{B}(T(v))$ has a structure of a $\mathfrak{g l}_{n}$-module with action of the generators of $\mathfrak{g l}_{n}$ given by the Gelfand-Tsetlin formulas.

$$
\begin{gathered}
E_{k, k+1}(T(v))=-\sum_{i=1}^{k}\left(\frac{\prod_{j=1}^{k+1}\left(v_{k i}-v_{k+1, j}\right)}{\prod_{j \neq i}^{k}\left(v_{k i}-v_{k j}\right)}\right) T\left(v+\delta^{k i}\right), \\
E_{k+1, k}(T(v))=\sum_{i=1}^{k}\left(\frac{\prod_{j=1}^{k-1}\left(v_{k i}-v_{k-1, j}\right)}{\prod_{j \neq i}^{k}\left(v_{k i}-v_{k j}\right)}\right) T\left(v-\delta^{k i}\right), \\
E_{k k}(T(v))=\left(k-1+\sum_{i=1}^{k} v_{k i}-\sum_{i=1}^{k-1} v_{k-1, i}\right) T(v) .
\end{gathered}
$$

where $\delta^{i j} \in \mathbb{C}^{\frac{n(n-1)}{2}}$ is defined by $\left(\delta^{i j}\right)_{i j}=1$ and all other $\left(\delta^{i j}\right)_{k \ell}$ are zero.

(ii) The $\mathfrak{g l}_{n}$-module $V(T(v))$ is a Gelfand-Tsetlin module with all non-zero Gelfand-Tsetlin multiplicities equal to 1.

Definition 3.5. For any Gelfand-Tsetlin tableau $T(v)$ of height $n$, we associate the following sets:

$$
\begin{aligned}
\Omega(T(v)) & :=\left\{(r, s, t): v_{r s}-v_{r-1, t} \in \mathbb{Z}\right\} \\
\Omega^{+}(T(v)) & :=\left\{(r, s, t): v_{r, s}-v_{r-1, t} \in \mathbb{Z}_{\geqslant 0}\right\} .
\end{aligned}
$$

Theorem 3.6 ([FGR15, Theorem 6.14]). Let $T(v)=T\left(v_{i j}\right)$ be a generic GelfandTsetlin tableau of height $n$. The simple subquotient of $V(T(v))$ which contains a tableau $T\left(v^{\prime}\right) \in \mathcal{B}(T(v))$ has the following basis of tableaux:

$$
\mathcal{I}\left(T\left(v^{\prime}\right)\right)=\left\{T\left(v^{\prime \prime}\right) \in \mathcal{B}(T(v)): \Omega^{+}\left(T\left(v^{\prime \prime}\right)\right)=\Omega^{+}\left(T\left(v^{\prime}\right)\right)\right\} .
$$

The action of $\mathfrak{g l}_{n}$ on this simple module is given by the classical Gelfand-Tsetlin formulas.

Corollary 3.7. If $\lambda=\left(\lambda_{1}, \ldots, \lambda_{n}\right)$ is a $\mathfrak{g l}_{n}$ weight such that $\lambda_{i}-\lambda_{j} \notin \mathbb{Z}$ then, the Verma module $M(\lambda)$ is simple and admits a tableau realization as a subquotient of a generic module $V(T(v))$ with basis

$$
\mathcal{I}(T(v))=\left\{T\left(v^{\prime}\right) \in \mathcal{B}(T(v)): \Omega^{+}\left(T\left(v^{\prime}\right)\right)=\{(r, s, s): 1 \leqslant s \leqslant r \leqslant n\}\right\}
$$

where $c_{i}=\lambda_{i}-i+1$ and $T(v)$ is the generic tableau with entries $v_{r s}=c_{s}$.

\section{Admissible Generic modules in the PRINCIPAL NiLPOTENT ORBIT}

We set $G:=S_{n} \times S_{n-1} \times \cdots S_{1}$ and the $i$-th component of $\sigma \in G$ will be denoted by $\sigma[i]$. Note that for any $\sigma=(\sigma[n], \sigma[n-1], \ldots, \sigma[1]) \in G$ we have $V(T(v)) \simeq V(T(\sigma(v)))$. We immediately have

Lemma 4.1. Let $T(v)$ be any strongly generic Gelfand-Tsetlin tableau. There exists $\sigma \in G$ such that $\Omega(T(\sigma(v))) \subseteq\{(r, s, s): 1 \leqslant s \leqslant r \leqslant n\}$. 
In [Kho05] is constructed an embedding of $U\left(\mathfrak{g l}_{n}\right)$ into a certain localization $\mathcal{D}_{m}$ of the Weyl algebra $\mathcal{A}_{m}$, for $m=n(n+1) / 2$ with generators $\left\{x_{i j}, \partial_{i j}: 1 \leqslant j \leqslant\right.$ $i \leqslant n\}$ (for details see [Kho05, Theorem 3.2]) based on Gelfand-Tsetlin formulas. Denote by $\phi$ this embedding and $\mathcal{P}$ the polynomial algebra in variables $y_{i j}=\partial_{i j} x_{i j}$. This embedding essentially provides a tableaux realization. As an application of the construction of this embedding an alternative proof of Duflo's Theorem (which describes the annihilators of Verma modules c.f, §2.4) for $\mathfrak{g l}_{n}$ is given. In particular, the description of annihilators of generic Verma modules is given in ([Kho05, Theorem 4.1]) together with their explicit tableaux realization (Corollary 3.7). In order to describe annihilators of simple subquotients of strongly generic modules we will use a slight modification of the proof of Theorem 4.1 in [Kho05].

Theorem 4.2. Let $T(v)$ be any strongly generic Gelfand-Tsetlin tableau. For any simple subquotient $N$ of $V(T(v))$ we have

$$
A n n_{U\left(\mathfrak{g l}_{n}\right)} N=U\left(\mathfrak{g l}_{n}\right) A n n_{Z\left(\mathfrak{g l}_{n}\right)} N .
$$

Proof. By Lemma 4.1 we can assume without loss of generality that $\Omega(T(v)) \subseteq$ $\{(r, s, s): 1 \leqslant s \leqslant r \leqslant n\}$. Let $N$ be a simple subquotient of $V(T(v))$, by Theorem 3.6, there exist $A \subseteq \Omega(T(v))$ such that $N$ is spanned by the set of tableaux $\mathcal{I}(N)=$ $\left\{T(w) \in \mathcal{B}(T(v)): \Omega^{+}(T(w))=A\right\}$.

Let $I=U\left(\mathfrak{g l}_{n}\right) A n n_{Z\left(\mathfrak{g l}_{n}\right)} N$. It is clear that $I \subseteq A n n_{U\left(\mathfrak{g l}_{n}\right)} N$. To prove the opposite inclusion, take some $u \in U\left(\mathfrak{g l}_{n}\right)$ such that $u N=0$. The image of $u$ under the inclusion $\phi$ can be written as $\phi(u)=\sum_{k=1}^{K} D_{k} f_{k}$ for some $K \in \mathbb{Z}_{\geqslant 0}$, $f_{k}$ in certain localization $\tilde{\mathcal{P}}$ of $\mathcal{P}$ and $D_{k}=\prod_{i=1}^{n-1} \prod_{j=1}^{i} \partial_{i j}^{n_{i j k}}$ with $n_{i j k} \in \mathbb{Z}$. For $w \in\left\{v+z: z \in \mathbb{Z}^{\frac{n(n-1)}{2}}\right\}$, let $I_{w} \subset \mathcal{P}$ be the ideal of $\mathcal{P}$ generated by $y_{i j}-w_{i j}$, for $1 \leqslant j \leqslant i \leqslant n$. One gets that $I_{w} \tilde{\mathcal{P}}$ is a proper maximal ideal of $\tilde{\mathcal{P}}$ for any $w$ such that $T(w) \in B$. For each rational function $f \in \tilde{\mathcal{P}}$ and $T(w) \in B$ define the evaluation $f(w)$ as the image of $f$ under the canonical projection $\tilde{\mathcal{P}} \rightarrow \tilde{\mathcal{P}} / I_{w} \tilde{\mathcal{P}} \simeq \mathbb{C}$. Let

$$
\begin{gathered}
L=\max \left\{\left|n_{i j k}\right|: 1 \leqslant k \leqslant K, 1 \leqslant i \leqslant n, 1 \leqslant j \leqslant i\right\}, \\
B=\left\{T(w) \in \mathcal{I}(N):\left|w_{r s}-w_{r-1, s}\right|>L, \text { for any }(r, s, s) \in \Omega(T(v))\right\}
\end{gathered}
$$

By construction, one easily obtains that for all $T(w) \in B$ the action of $u$ on $T(w)$ is given by

$$
u T(w)=\sum_{k=1}^{K} f_{k}(w) T\left(w+\sum_{i=1}^{n-1} \sum_{j=1}^{i} n_{i j k} \delta^{i j}\right) .
$$

Due to the choice of $T(w) \in B$ we have $T\left(w+\sum_{i=1}^{n-1} \sum_{j=1}^{i} n_{i j k} \delta^{i j}\right) \in \mathcal{I}(N)$ for any $k$, which implies $u T(w)=0$ if and only if $f_{k} \in I_{w} \tilde{\mathcal{P}}$ for all $1 \leqslant k \leqslant K$. Finally, by using just the fact that the module is generic, it is shown in ([Kho05, Theorem 4.1]) that $f_{k} \in I_{w} \tilde{\mathcal{P}}$ for all $1 \leqslant k \leqslant K$ implies $u \in I$.

4.1. Admissible generic Gelfand-Tsetlin modules in the principal nilpotent orbit for $\mathfrak{s l}_{n}$. Let $k$ be an admissible number for $\widehat{\mathfrak{s l}}_{n}$, so that

$$
k+n=p / q, \quad p \geqslant n, q \geqslant 1,(p, q)=1 .
$$


By Proposition 2.6, an element of $\left[\overline{\operatorname{Pr}}_{k}{ }^{\text {prin }}\right.$ ] for $\mathfrak{g}=\mathfrak{s l}_{n}$ is represented by the weight of the form

$$
\lambda^{\prime}=\lambda-\frac{p}{q}(\mu+\rho)=\left(\lambda_{1}-\frac{p}{q}\left(\mu_{1}+1\right), \lambda_{2}-\frac{p}{q}\left(\mu_{2}+1\right), \ldots, \lambda_{n-1}-\frac{p}{q}\left(\mu_{n-1}+1\right)\right),
$$

where $\lambda_{i}, \mu_{i} \in \mathbb{Z}_{\geqslant 0}$ are such that $\lambda_{1}+\ldots+\lambda_{n-1} \leqslant p-n, \mu_{1}+\ldots+\mu_{n-1} \leqslant q-n$. (The set $\left[\overline{\operatorname{Pr}}_{k}^{\mathbb{O}_{\text {prin }}}\right]$ is empty if $q<n$.)

$\mathrm{A} \mathfrak{g l}_{n}$-weight corresponding to $\lambda^{\prime}$ is $\left(b_{1}+b, \ldots, b_{n}+b\right)$, where for $1 \leqslant k \leqslant n-1$, we take $b_{i}:=\sum_{k=i}^{n-1}\left(\lambda_{k}-\frac{p}{q}\left(\mu_{k}+1\right)\right), b_{n}=0$ and $b=-\frac{1}{n} \sum_{i=1}^{n} b_{i}$. Note that for any $i<j, b_{i}-b_{j}=\sum_{k=i}^{j-1}\left(\lambda_{k}-\frac{p}{q}\left(\mu_{k}+1\right)\right) \notin \mathbb{Z}$. In particular, the corresponding simple highest weight module associated with $\lambda^{\prime}$ admits a tableaux realization as a subquotient of $V(T(v))$ with the strongly generic $T(v)$, where $v_{i j}=b_{j}+b-j+1$ for any $1 \leqslant j \leqslant i \leqslant n$.

The highest weight modules with the same annihilator as $L\left(\lambda^{\prime}\right)$ are all of the form $L\left(w \circ \lambda^{\prime}\right)$ with $w$ an element of the Weyl group. All these highest weight modules are simple Verma modules associated with some $\mathfrak{g l}_{n}$-weight of the form $\left(a_{1}, a_{2}, \ldots, a_{n}\right)$ satisfying $a_{i}-a_{j} \notin \mathbb{Z}$ for any $i \neq j$. Thus, in order to describe all simple GelfandTsetlin modules with the same annihilator as $L\left(\lambda^{\prime}\right)$ it is enough to describe simple Gelfand-Tsetlin modules with the same annihilator as a generic Verma module obtained by a tableaux realization as a subquotient of the module $V(T(v))$ with a strongly generic tableau $T(v)$ with entries $v_{i j}:=c_{j}$ for any $1 \leqslant j \leqslant i \leqslant n$.

Hence, Theorem 4.2 immediately implies

Corollary 4.3. Let $\lambda^{\prime}$ be an admissible $\mathfrak{s l}_{n}$-weight of level $k$ and $\left(a_{1}, \ldots, a_{n}\right)$ any corresponding $\mathfrak{g l}_{n}$-weight. Then any simple subquotient of a strongly generic module $\left\{V(T(v)): v_{n i}=a_{i}-i+1\right\}$ is a simple admissible Gelfand-Tsetlin module in the principal orbit. Moreover, any simple admissible generic Gelfand-Tsetlin module of level $k$ in the principal nilpotent orbit with central character determined by the $\mathfrak{g l}_{n^{-}}$ highest weight $\left(a_{1}, \ldots, a_{n}\right)$ is isomorphic to a subquotient of some strongly generic module $\left\{V(T(v)): v_{n i}=a_{i}-i+1\right\}$.

Now applying Theorem 3.6 we obtain a complete classification of all simple admissible generic Gelfand-Tsetlin modules of level $k$ in the principal orbit together with their explicit construction via the Gelfand-Tsetlin formulas.

Remark 4.4. - We observe that there exist simple generic Gelfand-Tsetlin modules which are not in the principal orbit.

- Among the modules described in Corollary 4.3 there are those with finite weight multiplicities. Such modules are either highest weight modules or induced from an $\mathfrak{s l}_{2}$-subalgebra. The latter modules will be also discussed in Theorem 6.5.

\section{Classification of simple admissible Gelfand-Tsetlin $\mathfrak{s l}_{3}$-Modules}

Let $k$ be an admissible number for $\mathfrak{g}=\mathfrak{s l}_{3}$, so that

$$
k+3=p / q, \quad p \geqslant 3, q \geqslant 1,(p, q)=1 .
$$

In this section we classify all simple Gelfand-Tsetlin admissible $\mathfrak{s l}_{3}$-module of an admissible level $k$. Using the classification of simple Gelfand-Tsetlin $\mathfrak{s l}_{3}$-modules 
[FGR], where explicit basis and the action of the generators of $\mathfrak{s l}_{3}$ are given, we are able to present explicitly all simple Gelfand-Tsetlin modules with necessary annihilator.

5.1. Singular Gelfand-Tsetlin $\mathfrak{s l}_{3}$-modules. Let $v_{r s}, 1 \leqslant s \leqslant r \leqslant 3$ complex numbers, $v=\left(v_{31}, v_{32}, v_{33}, v_{21}, v_{22}, v_{11}\right)$ and $w=(m, n, k):=\left(m_{21}, m_{22}, m_{11}\right) \in \mathbb{Z}^{3}$. We will denote by $T(v)$ and $T(v+w)$ the following tableaux:

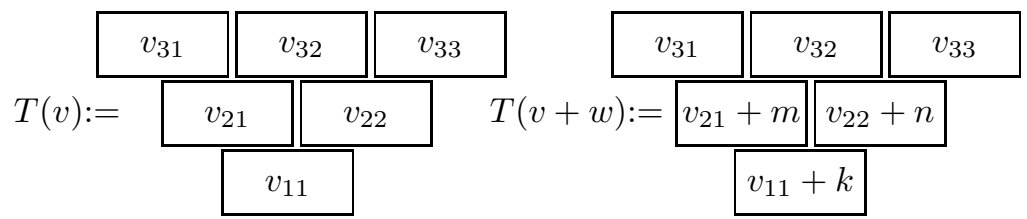

Also, set $\mathcal{B}(T(v)):=\left\{T(v+w): w \in \mathbb{Z}^{3}\right\}$

Definition 5.1. If $T\left(v^{\prime}\right)$ is any Gelfand-Tsetlin tableau from the set $\mathcal{B}(T(v))$. Then

(i) $T\left(v^{\prime}\right)$ is singular, if $v_{21}^{\prime}-v_{22}^{\prime} \in \mathbb{Z}$.

(ii) $T\left(v^{\prime}\right)$ is critical, if $v_{21}^{\prime}-v_{22}^{\prime}=0$.

We will follow [FGR] to construct an explicit basis of tableaux for simple singular Gelfand-Tsetlin $\mathfrak{s l}_{3}$-modules starting with a critical tableau.

From now on we will denote by $T(\bar{v})$ a fixed critical tableau and by $T(v)$ a generic tableau. Denote by $\tau$ the transposition $(1,2)$. We formally introduce a new tableau $\mathcal{D} T(\bar{v}+w)$ for every $w \in \mathbb{Z}^{3}$ subject to the relations $\mathcal{D} T(\bar{v}+w)+\mathcal{D} T(\bar{v}+\tau(w))=0$. We call $\mathcal{D} T(\bar{v}+w)$ the derivative Gelfand-Tsetlin tableau associated with $w$.

Definition 5.2. Set $V(T(\bar{v}))$ to be the vector space spanned by the set of tableaux $\left\{T(\bar{v}+w), \mathcal{D} T(\bar{v}+w) \mid w \in \mathbb{Z}^{3}\right\}$, subject to the relations $T(\bar{v}+w)=T(\bar{v}+\tau(w))$ and $\mathcal{D} T(\bar{v}+w)+\mathcal{D} T(\bar{v}+\tau(w))=0$. Choose a basis of $V(T(\bar{v}))$ to be the set $\left\{T(\bar{v}+w), \mathcal{D} T\left(\bar{v}+w^{\prime}\right): w, w^{\prime} \in \mathbb{Z}^{3}\right.$ and $\left.w_{21}-w_{22} \in \mathbb{Z}_{\geqslant 0}, w_{21}^{\prime}-w_{22}^{\prime} \in \mathbb{Z}_{<0}\right\}$.

For $v=\left(v_{31}, v_{32}, v_{33}, v_{21}, v_{22}, v_{11}\right)$ and a rational function $f$ on variables $v_{r s}$ which is smooth on the hyperplane $v_{21}-v_{22}=0$, define a linear map

$$
\mathcal{D}^{\bar{v}}(f T(v+z))=\mathcal{D}^{\bar{v}}(f) T(\bar{v}+z)+f(\bar{v}) \mathcal{D} T(\bar{v}+z),
$$

where $\mathcal{D}^{\bar{v}}(f)=\frac{1}{2}\left(\frac{\partial f}{\partial v_{21}}-\frac{\partial f}{\partial v_{22}}\right)(\bar{v})$.

Theorem 5.3 ([FGR]). For any critical tableau $T(\bar{v})$, the vector space $V(T(\bar{v}))$ together with the action of $\mathfrak{g l}_{3}$ given by

$$
\begin{aligned}
E_{r s}(T(\bar{v}+z)) & =\mathcal{D}^{\bar{v}}\left(\left(v_{21}-v_{22}\right) E_{r s}(T(v+z))\right) \\
\left.E_{r s}\left(\mathcal{D} T\left(\bar{v}+z^{\prime}\right)\right)\right) & =\mathcal{D}^{\bar{v}}\left(E_{r s}\left(T\left(v+z^{\prime}\right)\right)\right),
\end{aligned}
$$

for all $z, z^{\prime} \in \mathbb{Z}^{3}$ with $z^{\prime} \neq \tau\left(z^{\prime}\right)$, has a structure of a Gelfand-Tsetlin module over $\mathfrak{g l}_{3}$. Here, the action $E_{r s}(T(v+z))$ is given by the classical Gelfand-Tsetlin formulas as in the generic case.

Remark 5.4. This construction can be extended to $\mathfrak{g l}_{n}$ by considering GelfandTsetlin tableaux $T(v)$ with exactly one singularity (i.e. when exists a unique $(k, i, j)$ such that $\left.v_{k i}-v_{k j} \in \mathbb{Z}\right)$. Such modules were studied in [FGR16]. 
5.2. Construction of simple admissible Gelfand-Tsetlin modules for $\mathfrak{s l}_{3}$. In this subsection we will present explicitly simple subquotiens of $V(T(v)$ ) (respectively, of $V(T(\bar{v}))$ ) for generic $v$ (respectively, for critical $\bar{v}$ ) that have the same annihilator as admissible highest weight modules $L(\lambda)$.

In order to simplify the description we will use the following notation:

$$
\operatorname{Tab}(w):= \begin{cases}T(v+w), & \text { if } v \text { is generic, } \\ T(\bar{v}+w), & \text { if } \bar{v} \text { is critical and } w_{k i}-w_{k j} \leqslant 0, \\ \mathcal{D} T(\bar{v}+w), & \text { if } \bar{v} \text { is critical and } w_{k i}-w_{k j}>0 .\end{cases}
$$

If $D_{1}, \ldots, D_{k}$ are some sets of inequalities we denote

$$
L\left(D_{1} \cup \ldots \cup D_{k}\right)=\operatorname{span}\left\{\operatorname{Tab}(z) \mid z \text { satisfies one of } D_{1}, \ldots, D_{k}\right\} \text {. }
$$

Twisted localization. Let $U=U\left(\mathfrak{s l}_{3}\right)$. We recall the definition of the localization functor on $U$-modules. For details we refer the reader to [Deo80] and [Mat00].

For every $\alpha \in \Delta$ we fix an $\mathfrak{s l}(2)$-triple $\left(e_{\alpha}, f_{\alpha}, h_{\alpha}\right)$, i.e. $e_{\alpha} \in \mathfrak{g}_{\alpha}, f_{\alpha} \in \mathfrak{g}_{-\alpha}$ and $\left[e_{\alpha}, f_{\alpha}\right]=h_{\alpha}$. Since ad $f_{\alpha}$ acts locally finitely on $U$, the multiplicative set $\mathbf{F}_{\alpha}:=$ $\left\{f_{\alpha}^{n} \mid n \in \mathbb{Z}_{\geqslant 0}\right\} \subset U$ satisfies Ore's localizability conditions. Denote by $\mathcal{D}_{\alpha} U$ the localization of $U$ relative to $\mathbf{F}_{\alpha}$. If $M$ is a weight module the denote by $\mathcal{D}_{\alpha} M$ the $\alpha$-localization of $M$, defined as $\mathcal{D}_{\alpha} M=\mathcal{D}_{\alpha} U \otimes_{U} M$. If $f_{\alpha}$ is injective on $M$, then $M$ can be naturally viewed as a submodule of $\mathcal{D}_{\alpha} M$.

For $a \in \mathbb{C}$ and $u \in \mathcal{D}_{\alpha} U$ set

$$
\Theta_{a}(u):=\sum_{i \geqslant 0}\left(\begin{array}{c}
a \\
i
\end{array}\right)\left(\operatorname{ad} f_{\alpha}\right)^{i}(u) f_{\alpha}^{-i}
$$

where $\left(\begin{array}{c}a \\ i\end{array}\right)=\frac{a(a-1) \ldots(a-i+1)}{i !}$. For a $\mathcal{D}_{\alpha} U$-module $M$ denote by $\Phi_{\alpha}^{a} M$ the $\mathcal{D}_{\alpha} U$ module $M$ twisted by the action

$$
u \cdot v^{a}:=\left(\Theta_{a}(u) \cdot v\right)^{a},
$$

where $u \in \mathcal{D}_{\alpha} U, v \in M$, and $v^{a}$ stands for the element $v$ considered as an element of $\Phi_{\alpha}^{a} M$. We set $f_{\alpha}^{a} \cdot v:=v^{-a}$ in $\Phi_{\alpha}^{-a} M, a \in \mathbb{C}$.

The following lemma is standard.

Lemma 5.5. Let $M$ be $a \mathcal{D}_{\alpha} U$-module, $v \in M, u \in \mathcal{D}_{\alpha} U$ and $a, b \in \mathbb{C}$. Then

(i) $\Phi_{\alpha}^{a} M \simeq M$ whenever $a \in \mathbb{Z}$.

(ii) $\Phi_{\alpha}^{a}\left(\Phi_{\alpha}^{b} M\right) \simeq \Phi_{\alpha}^{a+b} M$ and, consequently, $\Phi_{\alpha}^{a} \circ \Phi_{\alpha}^{-a} M \simeq \Phi_{\alpha}^{-a} \circ \Phi_{\alpha}^{a} M \simeq M$.

(iii) $f_{\alpha}^{a} \cdot\left(f_{\alpha}^{b} \cdot v\right)=f_{\alpha}^{a+b} \cdot v$.

(iv) $f_{\alpha}^{a} \cdot\left(u \cdot\left(f_{\alpha}^{-a} \cdot v\right)\right)=\Theta_{a}(u) \cdot v$.

Let $M$ be any Gelfand-Tsetlin module and $\Phi_{\alpha}^{a} M$ the twisted localization of $M$ for some $\alpha \in \Delta$ and $a \in \mathbb{C}$. It is clear that $A n n_{U}(M)=A n n_{U}\left(\Phi_{\alpha}^{a} M\right)$.

5.3. Minimal orbit. By Proposition 2.11, elements of $\left[\overline{\operatorname{Pr}}_{k}^{\mathbb{O}_{\text {min }}}\right]$ are represented by $\mathfrak{s l}_{3}$-weights of the form

$$
\lambda-\frac{a p}{q} \varpi_{1}=\left(\lambda_{1}-\frac{a p}{q}, \lambda_{2}\right),
$$

where $a, \lambda_{1}, \lambda_{2} \in \mathbb{Z}_{\geqslant 0}$ are such that $\lambda_{1}+\lambda_{2} \leqslant p-3$ and $1 \leqslant a \leqslant q-1$.

First we identify simple highest weight modules whose annihilator coincides with the one of $L\left(\lambda_{1}-\frac{a p}{q}, \lambda_{2}\right)$. They are the following modules:

(i) $L\left(\lambda_{1}-\frac{a p}{q}, \lambda_{2}\right)$. 
(ii) $L\left(\lambda_{2}, \frac{a p}{q}-\lambda_{1}-\lambda_{2}-3\right)$.

(iii) $L\left(\frac{a p}{q}-\lambda_{1}-2, \lambda_{1}+\lambda_{2}-\frac{a p}{q}+1\right)$.

Note that these highest weight modules have bounded weight multiplicities of dimension at most $t=\lambda_{2}+1$.

Next we describe simple Gelfand-Tsetlin modules with the same annihilator as highest weight modules above.

Fix $c:=\frac{\lambda_{2}+2 \lambda_{1}-\frac{2 a p}{q}}{3}, x:=\frac{\lambda_{2}-\lambda_{1}+\frac{a p}{q}}{3}-1, t=\lambda_{2}+1$ (note that $c-x \notin \mathbb{Z}$ ) and consider any $y, z \in \mathbb{C}$ such that $\{c-z, x-z, c-y, x-y, z-y\} \cup \mathbb{Z}=\emptyset$.

Theorem 5.6. The following modules constitute a complete list of non-isomorphic simple admissible Gelfand-Tsetlin modules of level $k$ in the minimal orbit $\mathbb{O}_{\text {min }}$. All these modules have finite dimensional weight spaces.

(i) The following subquotients of the generic module $V(T(v))$ with $v=(c, x, x-$ $t, c, x, c)$

$L_{1}:=L\left(\begin{array}{c}-t<n \leqslant 0 \\ m \leqslant 0 \\ k \leqslant m\end{array}\right) ; \quad L_{2}=L\left(\begin{array}{c}m \leqslant 0 \\ -t<n \leqslant 0 ; \\ m<k\end{array}\right) ; \quad L_{3}=L\left(\begin{array}{c}0<m \\ -t<n \leqslant 0 ; \\ k \leqslant m\end{array}\right) ; \quad L_{4}=L\left(\begin{array}{c}0<m \\ -t<n \leqslant 0 ; \\ m<k\end{array}\right)$

These modules are highest weight modules (with respect to some Borel subalgebra) with weight multiplicities bounded by $t$.

(ii) Subquotients of the generic module $V\left(T\left(v_{1}\right)\right)$ with $v_{1}=(c, x, x-t, c, x, z)$

$$
L_{5}=L\left(\begin{array}{c}
-t<n \leqslant 0 \\
m \leqslant 0
\end{array}\right) ; \quad L_{6}=L\left(\begin{array}{c}
-t<n \leqslant 0 \\
0<m
\end{array}\right)
$$

Both $L_{5}$ and $L_{6}$ are $\mathfrak{s l}_{2}$-induced modules (with respect to opposite parabolic subalgebras where $\mathfrak{s l}_{2}$ is generated by $\left.E_{12}, E_{21}\right)$. Weight multiplicities of these modules are bounded by $t$.

(iii) Generic cuspidal module with all weight multiplicities $t$ which is a subquotient of $V\left(T\left(v_{2}\right)\right)$ with $v_{2}=(c, x, x-t, y, x, z)$ :

$$
L_{7}=L(-t<n \leqslant 0)
$$

(iv) $\mathfrak{s l}_{2}$-induced modules (with respect to opposite parabolic subalgebras where $\mathfrak{s l}_{2}$ is generated by $\left.E_{13}, E_{31}\right)$ with weight multiplicities bounded by $t$ which are subquotients of the generic module $V\left(T\left(v_{3}\right)\right)$ with $v_{3}=(c, x, x-t, z, x, z)$ :

$$
L_{8}=L\left(\begin{array}{c}
-t<n \leqslant 0 \\
m<k
\end{array}\right) ; \quad L_{9}=L\left(\begin{array}{c}
-t<n \leqslant 0 \\
k \leqslant m
\end{array}\right)
$$

(v) The following subquotients of the generic module $V(T(u))$ with $u=(c, x, x-$ $t, x, c, x)$ :

$$
L_{10}=L\left(\begin{array}{c}
-t<m \leqslant 0 \\
n \leqslant 0 \\
k \leqslant m
\end{array}\right) ; L_{11}=L\left(\begin{array}{c}
-t<m \leqslant 0 \\
n \leqslant 0 \\
m<k
\end{array}\right) ; L_{12}=L\left(\begin{array}{c}
-t<m \leqslant 0 \\
0<n \\
k \leqslant m
\end{array}\right) ; L_{13}=L\left(\begin{array}{c}
-t<m \leqslant 0 \\
0<n \\
m<k
\end{array}\right)
$$

These are highest weight modules (with respect to some Borel subalgebra) with weight multiplicities bounded by $t$.

(vi) $\mathfrak{s l}_{2}$-induced modules (with respect to opposite parabolic subalgebras where $\mathfrak{s l}_{2}$ is generated by $\left.E_{23}, E_{32}\right)$ with weight multiplicities bounded by $t$ which are subquotients of the generic module $V\left(T\left(u_{1}\right)\right)$ with $u_{1}=(c, x, x-t, x, y, x)$

$$
L_{14}=L\left(\begin{array}{c}
-t<m \leqslant 0 \\
k \leqslant m
\end{array}\right) ; \quad L_{15}=L\left(\begin{array}{c}
-t<m \leqslant 0 \\
m<k
\end{array}\right)
$$


(vii) Subquotients of the singular module $V(T(\bar{v}))$ with $\bar{v}=(c, x, x-t, x, x, x)$ :

$$
\begin{aligned}
& L_{16}=L\left(\begin{array}{l}
-t<n \leqslant 0 \\
m \leqslant-t \\
m<k \leqslant n
\end{array}\right) ; L_{18}:=L\left(\left\{\begin{array} { c } 
{ m \leqslant n } \\
{ - t < m \leqslant 0 } \\
{ 0 < n } \\
{ k \leqslant m }
\end{array} \quad \bigcup \left\{\begin{array}{c}
m \leqslant n \\
-t<m \leqslant 0 \\
n \leqslant 0 \\
k \leqslant n
\end{array} \quad \bigcup\left\{\begin{array}{c}
n<m \\
-t<m \leqslant 0 \\
k \leqslant n
\end{array}\right)\right.\right.\right. \\
& L_{17}:=L\left(\begin{array}{c}
-t<m \leqslant 0 \\
0<n \\
m<k \leqslant n
\end{array}\right) ; \quad L_{19}:=L\left(\left\{\begin{array} { c } 
{ m \leqslant n } \\
{ - t < m \leqslant 0 } \\
{ n < k }
\end{array} \quad \bigcup \left\{\begin{array}{c}
n<m \\
-t<m \leqslant 0 \\
n \leqslant-t \\
m<k
\end{array} \quad \bigcup\left\{\begin{array}{c}
n<m \\
-t<m \leqslant 0 \\
-t<n \leqslant 0 \\
n<k
\end{array}\right)\right.\right.\right.
\end{aligned}
$$

These are highest weight modules (with respect to some Borel subalgebra) with weight multiplicities bounded by $t$. Modules $L_{16}$ and $L_{17}$ have all 1dimensional Gelfand-Tsetlin subspaces.

(viii) Cuspidal module with weight multiplicities $t$, which is a subquotient of the singular module $V\left(T\left(\bar{v}_{1}\right)\right)$ with $\bar{v}_{1}=(c, x, x-t, x, x, z)$,

$$
L_{20}=L\left(\left\{\begin{array}{c}
m \leqslant n \\
-t<m \leqslant 0
\end{array} \bigcup\left\{\begin{array}{c}
n<m \\
-t<n \leqslant 0
\end{array}\right)\right.\right.
$$

Proof. Associated with the $\mathfrak{s l}_{3}$-weight $\left(\lambda_{1}-\frac{2 a p}{q}, \lambda_{2}\right)$ we have the $\mathfrak{g l}_{3}$-weight

$$
\left(\frac{\lambda_{2}+2 \lambda_{1}-\frac{2 a p}{q}}{3}, \frac{\lambda_{2}-\lambda_{1}+\frac{a p}{q}}{3}, \frac{-2 \lambda_{2}-\lambda_{1}+\frac{a p}{q}}{3}\right) .
$$

The module $L\left(\lambda_{1}-\frac{a p}{q}, \lambda_{2}\right)$ can be realized as a subquotient of the generic GelfandTsetlin module $V(T(v))$ where

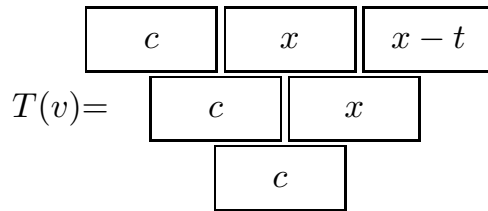

A basis for this module is given by the set of tableaux $L_{1}$. Using localization functor we identify another three simple subquotients of $V(T(v))$ with the same annihilator: $L_{2}, L_{3}$ and $L_{4}$. Now applying twisted localization functor with respect to $E_{21}$, we obtain two simple subquotients of the generic module $V\left(T\left(v_{1}\right)\right)$ where

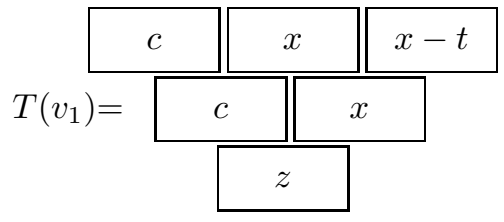

The corresponding basis are $L_{5}$ and $L_{6}$. Using twisted localization functor with respect to $E_{32}$ we obtain a cuspidal module with all $t$-dimensional weight multiplicities. This module can be realized as a subquotient of the generic Gelfand-Tsetlin module $V\left(T\left(v_{2}\right)\right)$, where

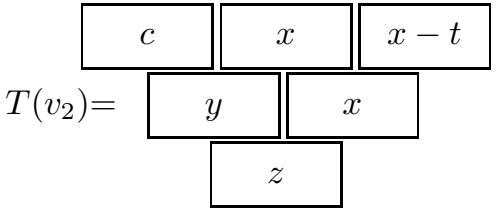


A basis is given by $L_{7}$. Now, applying twisted localization functor with respect to $E_{31}$ we obtain two simple subquotients of the generic module $V\left(T\left(v_{3}\right)\right)$, where

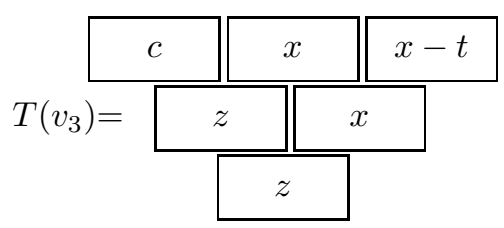

Their bases are $L_{8}$ and $L_{9}$.

Associated with the $\mathfrak{s l}_{3}$-weight $\left(\lambda_{2}, \frac{a p}{q}-\lambda_{1}-\lambda_{2}-3\right)$ we have the $\mathfrak{g l}(3)$-weight

$$
\left(\frac{\lambda_{2}-\lambda_{1}+\frac{a p}{q}}{3}, \frac{\lambda_{2}+2 \lambda_{1}-\frac{2 a p}{q}}{3}, \frac{-2 \lambda_{2}-\lambda_{1}+\frac{a p}{q}}{3}\right) .
$$

So, the highest weight module $L\left(\lambda_{2}, \frac{a p}{q}-\lambda_{1}-\lambda_{2}-3\right)$ has a tableaux realization as a subquotient of the generic Gelfand-Tsetlin module $V(T(u))$ where

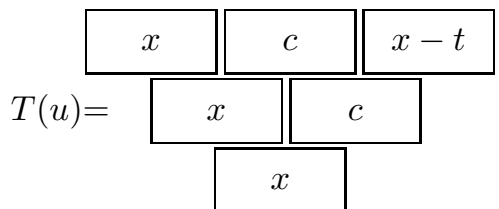

A basis for this module is given by the set of tableaux $L_{10}$. Also, the module $V(T(u))$ contains three other simple subquotiens with the desired annihilator. Their bases are $L_{11}, L_{12}, L_{13}$.

Applying twisted localization functor with respect to $E_{32}$ we obtain modules that can be realized as a subquotient of the generic Gelfand-Tsetlin module $V\left(T\left(u_{1}\right)\right)$, where

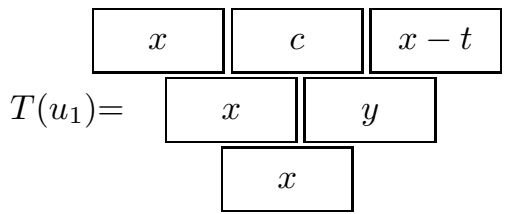

These two modules have explicit basis $L_{14}$ and $L_{15}$ respectively.

Finally, associated with the $\mathfrak{s l}_{3}$-weight $\left(\frac{a p}{q}-\lambda_{1}-2, \lambda_{1}+\lambda_{2}-\frac{a p}{q}+1\right)$ is the $\mathfrak{g l}(3)$-weight

$$
\left(\frac{\lambda_{2}-\lambda_{1}+\frac{a p}{q}}{3}, \frac{-2 \lambda_{2}-\lambda_{1}+\frac{a p}{q}}{3}, \frac{\lambda_{2}+2 \lambda_{1}-\frac{2 a p}{q}}{3}\right) .
$$

So, the module $L\left(\frac{a p}{q}-\lambda_{1}-2, \lambda_{1}+\lambda_{2}-\frac{a p}{q}+1\right)$ can be realized as a subquotient $L_{16}$ of the singular Gelfand-Tsetlin module $V(T(\bar{v}))$ where

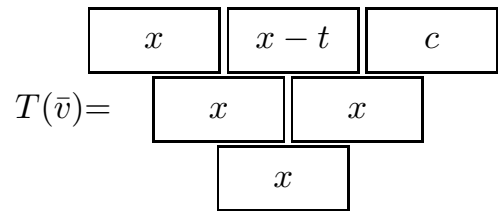


The module $V(T(\bar{v}))$ has another three simple subquotients with the same annihilator. Their bases are $L_{17}, L_{18}$ and $L_{19}$ respectively.

Applying the twisted localization functor with respect to $E_{21}$ we obtain a cuspidal module with $t$-dimensional weight multiplicities that can be realized as a subquotient of the singular Gelfand-Tsetlin module $V\left(T\left(\bar{v}_{1}\right)\right)$, where

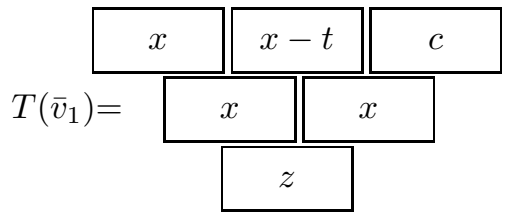

A basis for this module is given by $L_{20}$.

Remark 5.7. A complete list of non-isomorphic simple admissible Gelfand-Tsetlin modules in the minimal orbit is obtained when $q=2$ and $a=1$ in the theorem above (see Proposition 2.11). All other modules correspond to minimal nilpotent orbits in the closure of the principal orbit with $q \geqslant 3$.

5.4. Principal orbit $(q \geqslant 3)$. By Proposition 2.11 the elements of $\left[\overline{\operatorname{Pr}}_{k}^{\mathbb{Q}_{\text {prin }}}\right]$ are represented by $\mathfrak{s l}_{3}$-weights of the form

$$
\lambda^{\prime}=\lambda-\frac{p}{q}(\mu+\rho)=\left(\lambda_{1}-\frac{p}{q}\left(\mu_{1}+1\right), \lambda_{2}-\frac{p}{q}\left(\mu_{2}+1\right)\right),
$$

where $\lambda_{1}, \lambda_{2}, \mu_{1}, \mu_{2} \in \mathbb{Z}_{\geqslant 0}$ are such that $\lambda_{1}+\lambda_{2} \leqslant p-3, \mu_{1}+\mu_{2} \leqslant q-3$. Note that this set is non-empty if and only if $q \geqslant 3$. A $\mathfrak{g l}(3)$ weight associated with $\lambda^{\prime}$ is $\left(a_{1}, a_{2}, a_{3}\right)$, where $a_{1}=\frac{1}{3}\left(2 \lambda_{1}+\lambda_{2}-\frac{p}{q}\left(2 \mu_{1}+\mu_{2}+3\right)\right), a_{2}=$ $\frac{1}{3}\left(\lambda_{2}-\lambda_{1}-\frac{p}{q}\left(\mu_{2}-\mu_{1}\right)\right)$ and $a_{3}=-\frac{1}{3}\left(\lambda_{1}+2 \lambda_{2}-\frac{p}{q}\left(\mu_{1}+2 \mu_{2}+3\right)\right)$. First we identify simple highest weight modules in the principal orbit, i.e. having the same annihilator as $L\left(\lambda^{\prime}\right)$. They are all of the form $L\left(w \circ \lambda^{\prime}\right)$ with $w$ an element of the Weyl group. All those highest weight modules are simple Verma modules associated with some $\mathfrak{g l}_{3}$-weight of the form $(a, b, c)$ satisfying $\{a-b, a-c, b-c\} \cap \mathbb{Z}=\emptyset$. Hence, it is sufficient to describe simple Gelfand-Tsetlin modules in the same orbit with simple generic Verma module which is a subquotient of $V(T(v))$, where $v=(x, y, z, x, y, x)$ and $\{x-y, x-z, y-z\} \cap \mathbb{Z}=\emptyset$.

Note that in this orbit we will have both generic and singular Gelfand-Tsetlin modules.

Theorem 5.8. The following modules constitute a complete list of non-isomorphic infinite-dimensional simple admissible Gelfand-Tsetlin $\mathfrak{s l}_{3}$-modules of level $k$ with denominator $q \geqslant 3$.

(i) Simple admissible modules described in Theorem 5.6 when $q \geqslant 3$.

(ii) Simple generic admissible Gelfand-Tsetlin modules. Any such module is isomorphic to a simple subquotient of $V\left(T\left(v^{\prime}\right)\right)$ where $T\left(v^{\prime}\right)$ is a strongly generic tableau with top row $(x, y, z):=\left(a_{1}, a_{2}-1, a_{3}-2\right)$ (see Theorem 3.6 and Corollary 4.3).

(iii) Simple singular admissible Gelfand-Tsetlin modules which are isomorphic to one of the following modules: 
- Subquotients of $V(T(\bar{v}))$, where $\bar{v}=(x, y, z, x, x, x)$ :

$$
\begin{gathered}
L_{1}=L\left(\left\{\begin{array}{l}
m \leqslant 0 \\
0<n \\
k \leqslant m
\end{array} \quad \bigcup\left\{\begin{array}{l}
m \leqslant 0 \\
n \leqslant 0 \\
k \leqslant n
\end{array}\right) ; L_{2}=L\left(\left\{\begin{array}{l}
0<m \\
0<n \\
n<k
\end{array} \quad \bigcup\left\{\begin{array}{l}
0<m \\
n \leqslant 0 \\
m<k
\end{array}\right)\right.\right.\right.\right. \\
L_{3}=L\left(\begin{array}{l}
m \leqslant 0 \\
n<k
\end{array}\right) ; L_{4}=L\left(\left\{\begin{array}{l}
0<m \\
k \leqslant n
\end{array}\right) ; \quad L_{5}=L\left(\begin{array}{l}
m \leqslant 0<n \\
m<k \leqslant n
\end{array}\right)\right.
\end{gathered}
$$

Here, $L_{1}, L_{2}, L_{3}, L_{4}$ are $\mathfrak{s l}_{2}$-induced modules with unbounded weight multiplicities and $L_{5}$ is cuspidal with infinite weight multiplicities. Modules $L_{1}$ and $L_{2}$ are induced from $\mathfrak{s l}_{2}$ (with respect to opposite parabolic subalgebras where $\mathfrak{s l}_{2}$ is generated by $E_{23}, E_{32}$ ), while $L_{3}$ and $L_{4}$ are induced from $\mathfrak{s l}_{2}$ (with respect to opposite parabolic subalgebras where $\mathfrak{s l}_{2}$ is generated by $\left.E_{13}, E_{31}\right)$.

- Cuspidal modules with infinite weight multiplicities which are subquotients of $V\left(T\left(\bar{v}_{1}\right)\right)$ with $\bar{v}_{1}=(x, y, z, x, x, a)$ :

$$
L_{6}=L\left(\left\{\begin{array}{l}
m \leqslant n \\
m \leqslant 0
\end{array} \quad \bigcup\left\{\begin{array}{l}
n<m \\
m \leqslant 0
\end{array}\right) ; \quad L_{7}=L\left(\left\{\begin{array}{l}
m \leqslant n \\
0<m
\end{array} \quad \bigcup\left\{\begin{array}{l}
n<m \\
0<m
\end{array}\right)\right.\right.\right.\right.
$$

- Cuspidal modules with infinite weight multiplicities which are subquotients of $V\left(T\left(\bar{v}_{2}\right)\right)$ with $\bar{v}_{2}=(x, y, z, a, a, a)$ :

$$
L_{8}=L\left(\left\{\begin{array}{l}
m \leqslant n \\
k \leqslant n
\end{array} \bigcup\left\{\begin{array}{l}
n<m \\
k \leqslant n
\end{array}\right) ; \quad L_{9}=L\left(\left\{\begin{array}{l}
m \leqslant n \\
n<k
\end{array} \quad \bigcup\left\{\begin{array}{l}
n<m \\
n<k
\end{array}\right)\right.\right.\right.\right.
$$

- Cuspidal module with infinite weight multiplicities $V\left(T\left(\bar{v}_{3}\right)\right)$ with $\bar{v}_{3}=$ $(x, y, z, a, a, c)$.

Proof. The proof is based on the classification and explicit presentation of all simple Gelfand-Tsetlin $\mathfrak{s l}_{3}$-modules (see [FGR] $\S 7$ and $\S 8$ ).

(i) Follows by Proposition 2.11.

(ii) These modules are described in Corollary 4.3.

(iii) Set $a, c \in \mathbb{C}$ such that $\{x-a, y-a, z-a, c-a\} \cap \mathbb{Z}=\emptyset$. Applying the twisted localization functor with respect to $E_{32}$ with parameter $a$ to the Verma module generated by the tableau

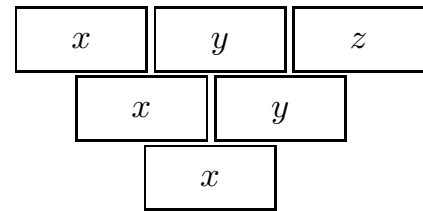

we obtain the singular module $V\left(T\left(\bar{v}_{0}\right)\right)$ where

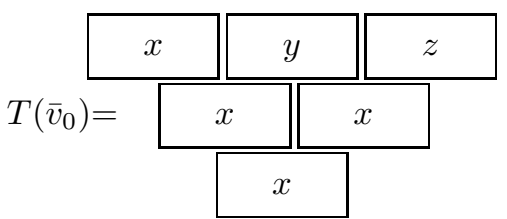

The module $V\left(T\left(\bar{v}_{0}\right)\right)$ has five simple subquotiens with the same annihilator. They correspond to the modules $L_{1}, L_{2}, L_{3}$ and $L_{4}$ (with unbounded 
but finite dimensional weight multiplicities) and a module with infinite dimensional weight spaces $L_{5}$. Applying twisted localization functor with respect to $E_{21}$ to the previous case, we obtain modules on the singular block $V\left(T\left(\bar{v}_{1}\right)\right)$ where:

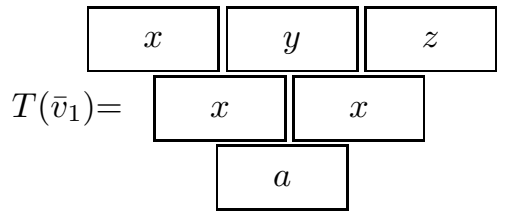

The module $V\left(T\left(\bar{v}_{1}\right)\right)$ has two simple subquotiens with the same annihilator, both of them having infinite weight multiplicities. They correspond to the modules $L_{6}$ and $L_{7}$. Consider

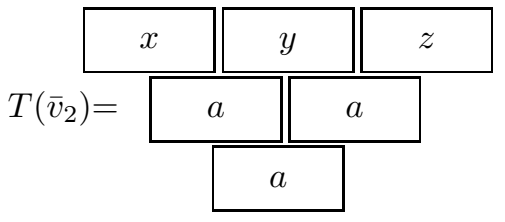

The singular module $V\left(T\left(\bar{v}_{2}\right)\right)$ has two simple subquotiens with infinite dimensional weight spaces corresponding to $L_{8}$ and $L_{9}$. Finally, consider

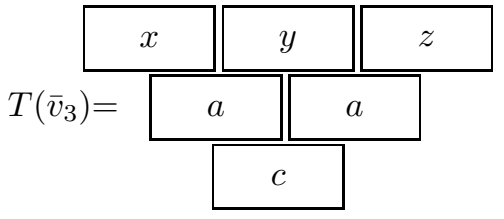

which is obtained by application on the twisted localization functor with respect to $E_{21}$ to the previous case The singular module $V\left(T\left(\bar{v}_{3}\right)\right)$ is simple with infinite dimensional weight multiplicities. It corresponds to $L_{10}$.

Remark 5.9. Modules $L_{1}, \ldots, L_{4}$ in Theorem 5.8 have unbounded finite weight multiplicities which grow linearly. They are isomorphic to the corresponding generalized Verma modules (induced from unbounded simple $s l(2)$-modules).

\section{Admissible $\mathfrak{s l}_{n}$-MODUles INDUCED FROM $\mathfrak{s l}_{k}$-MODULES}

Let $k$ be an admissible number for $\widehat{\mathfrak{s l}}_{n}$, so that

$$
k+n=p / q, \quad p \geqslant n, q \geqslant 1,(p, q)=1 .
$$

In this section we describe families of simple admissible $\mathfrak{g}=\mathfrak{s l}_{n}$-modules induced from simple $\mathfrak{s l}_{k}$-modules for $k=2,3$. If $M=L_{\mathfrak{p}}(\lambda, N)$ is admissible for some parabolic subalgebra $\mathfrak{p}$ with Levi subalgebra $\mathfrak{l}$ and nilradical $\mathfrak{m}$, then $M^{\mathfrak{m}} \simeq N$ and, hence, $N$ is admissible $\mathfrak{l}$-module by Theorem 2.12. Therefore, we need to consider $\mathfrak{s l}_{n}$-modules $M$ induced from simple admissible $\mathfrak{s l}_{k}$-modules.

We will provide a tableaux realization for admissible $\mathfrak{s l}_{n}$-modules induced from simple Gelfand-Tsetlin $\mathfrak{s l}_{k}$-modules in the principal orbit for $k=2,3$. 
Remark 6.1. We defined Gelfand-Tsetlin $\mathfrak{g l}_{n}$-modules in connection with the fixed Gelfand-Tsetlin subalgebra (which is maximal commutative) determined by a special chain of embeddings of $\mathfrak{g l}_{i}$ 's into each other (left upper corner inclusion). On the other hand, one can consider a different chain of embeddings starting from an arbitrary root of $\mathfrak{g l}_{n}$. This would produce a different maximal commutative subalgebra of $U\left(\mathfrak{g l}_{n}\right)$ and one could consider Gelfand-Tsetlin modules with respect to this subalgebra. But, it is clear, that all such chains of embeddings are conjugated by the Weyl group and, hence, the categories of corresponding Gelfand-Tsetlin modules are equivalent. Same holds for Gelfand-Tsetlin modules over $\mathfrak{s l}_{n}$. If $\mathfrak{s l}_{k}$ is a root subalgebra of $\mathfrak{s l}_{n}$ then there exists a parabolic subalgebra $\mathfrak{p}=\mathfrak{l} \oplus \mathfrak{n}$ with $\mathfrak{l}=\mathfrak{s l}_{k}+\mathfrak{h}$. Then for a Gelfand-Tsetlin $\mathfrak{s l}_{k}$-module $N$ with respect to certain choice of embeddings of $\mathfrak{s l}_{k}$, the induced module $M_{\mathfrak{p}}(\lambda, N)$ will be a Gelfand-Tsetlin module for a certain chain of embeddings of $\mathfrak{s l}_{n}$ which contains $\mathfrak{s l}_{k}$. This module in general may not be a Gelfand-Tsetlin module with respect to the Gelfand-Tsetlin subalgebra (left upper corner inclusion). But, due to the category equivalence mentioned above, we can always assume without loss of generality that $\mathfrak{s l}_{k}$ subalgebra belongs to the chain of the left upper corner inclusions and that $M_{\mathfrak{p}}(\lambda, N)$ is a Gelfand-Tsetlin module with respect to the Gelfand-Tsetlin subalgebra.

6.1. Construction of induced modules. Let $N$ be any simple $\mathfrak{s l}_{k}$-module ( $k=$ $2,3)$ with tableaux realization as a subquotient of a module $V(T(u))$ with generic top row (i.e. $u_{21}-u_{22} \notin \mathbb{Z}$ for $k=2$ and $\left\{u_{31}-u_{32}, u_{31}-u_{33}, u_{32}-u_{33}\right\} \cap \mathbb{Z}=\emptyset$ for $k=3)$.

$\mathfrak{s l}_{2}$-case. Set $v_{1}:=u_{21}$ and $v_{2}:=u_{22}$. Choose also $\left\{v_{i}\right\}_{i=3, \ldots, n}$ complex numbers such that $v_{i}-v_{j} \notin \mathbb{Z}$ for any $1 \leqslant i<j \leqslant n$. Let $T(v)$ be the Gelfand-Tsetlin tableau with entries

$$
v_{i j}= \begin{cases}v_{j}, & \text { if } i \geqslant 2 \\ u_{11}, & \text { if } i=1\end{cases}
$$

for $1 \leqslant j \leqslant i \leqslant n$.

Lemma 6.2. If $T(v)$ is the above Gelfand-Tsetlin tableau then there exist a subquotient $M$ of $V(T(v))$ isomorphic to an induced module $M_{\mathfrak{p}}(\lambda, N)$, where $\mathfrak{p}$ has the Levi subalgebra isomorphic to $\mathfrak{s l}_{2}$ and $N$ is cuspidal $\mathfrak{s l}_{2}$-module. Moreover, $M$ is simple if and only if $u_{21}-u_{11}, u_{22}-u_{11} \notin \mathbb{Z}$. It has a basis given by the set of tableaux $T(w)$ in $\mathcal{B}(T(v))$ such that $w_{r s}-w_{r-1, s} \in \mathbb{Z}_{\geqslant 0}$ for any $3 \leqslant r \leqslant n, 1 \leqslant s \leqslant r-1$.

Proof. By construction the module $V(T(v))$ is generic. The assertions follow from Theorems 3.4 and 3.6.

Denote by $M\left(N, v_{3}, \ldots, v_{n}\right)$ the induced module $M$ described in Lemma 6.2.

$\mathfrak{s l}_{3}$-case. Set $v_{1}:=u_{31}, v_{2}:=u_{32}$ and $v_{3}:=u_{33}$. Choose $\left\{v_{i}\right\}_{i=4, \ldots, n}$ complex numbers such that $v_{i}-v_{j} \notin \mathbb{Z}$ for any $1 \leqslant i<j \leqslant n$. Let $T(v)$ be the GelfandTsetlin tableau with entries

$$
v_{i j}= \begin{cases}v_{j}, & \text { if } i \geqslant 3 \\ u_{i j}, & \text { if } i \leqslant 2\end{cases}
$$

for $1 \leqslant j \leqslant i \leqslant n$.

Lemma 6.3. If $T(v)$ is the above Gelfand-Tsetlin tableau then there exists a subquotient $M$ of $V(T(v))$ isomorphic to an induced module $M_{\mathfrak{p}}(\lambda, N)$, where $\mathfrak{p}$ 
has the Levi subalgebra isomorphic to $\mathfrak{s l}_{3}$ and $N$ is cuspidal $\mathfrak{s l}_{3}$-module. If $v$ is generic then $M$ has a basis given by the set of tableaux $T(w)$ in $\mathcal{B}(T(v))$ such that $w_{r s}-w_{r-1, s} \in \mathbb{Z}_{\geqslant 0}$ for any $4 \leqslant r \leqslant n, 1 \leqslant s \leqslant r-1$. Moreover, $M$ is simple if and only if $u_{r s}-u_{r-1, t} \notin \mathbb{Z}$ for any $1 \leqslant s \leqslant r \leqslant 3$ and $1 \leqslant t \leqslant r-1$.

Proof. If $v$ is singular then the tableau $T(v)$ and the module $V(T(v))$ is 1-singular (see [FGR16] and Remark 5.4). If $v$ is generic then the module $V(T(v))$ is generic and the assertions follows from Theorems 3.4 and 3.6.

Denote by $M\left(N, v_{4}, \ldots, v_{n}\right)$ the induced module described in Lemma 6.3.

Remark 6.4. Set $N$ a simple $\mathfrak{s l}_{3}$-module.

(i) If $N$ is not cuspidal then $M\left(N, v_{4}, \ldots, v_{n}\right)$ is isomorphic to a Verma module or to a Generalized Verma module induced from $\mathfrak{s l}_{2}$.

(ii) If $N$ is cuspidal then all weight multiplicities of $M\left(N, v_{4}, \ldots, v_{n}\right)$ are infinite.

6.2. Admissible $\mathfrak{s l}_{2}$-induced modules in the principal orbit revisited. Any simple weight $\mathfrak{s l}_{2}$-module is a Gelfand-Tsetlin module and it admits a tableaux realization. Since we are interested in induced non-highest weight modules we can consider only cuspidal $\mathfrak{s l}_{2}$-modules. Any such module is a module in the principal nilpotent orbit of $\mathfrak{s l}_{2}$ and it is isomorphic to $V(T(u))$ for some generic $u$. In particular, for admissible $\mathfrak{s l}_{2}$-module $V(T(u))$ in the principal nilpotent orbit we have $u=\left(u_{21}, u_{22}, u_{11}\right)$ where $u_{21}-u_{22}-1=\lambda-\frac{p}{q}(\mu+1)$ for some $\lambda, \mu \in \mathbb{Z}_{\geqslant 0}$ satisfying $\lambda \leqslant p-2$ and $\mu \leqslant q-2$ with $p, q \in \mathbb{N},(p, q)=1, p, q \geqslant 2$. We say in this case that $V(T(u))$ (and its simple quotient which contains $T(u)$ ) is associated with the weight $\lambda-\frac{p}{q}(\mu+1)$. Induced module from a generic $\mathfrak{s l}_{2}$-module need not to be generic. In fact, there exist singular induced modules as we saw in the case of $\mathfrak{s l}_{3}$.

The following theorem gives a complete classification of all simple weight generic admissible $\mathfrak{s l}_{2}$-induced modules in the principal orbit.

Theorem 6.5. Let $N$ be a simple admissible cuspidal $\mathfrak{s l}_{2}$-module associated with the weight $\lambda^{\prime}=\lambda_{1}-\frac{p}{q}\left(\mu_{1}+1\right)$. If $\lambda_{1} \leqslant p-n, \mu_{1} \leqslant q-n$ and $p, q \geqslant n$ then for any choice of $\left\{\lambda_{i}, \mu_{i}\right\}_{i=2, \ldots, n-1} \subseteq \mathbb{Z}_{\geqslant 0}$ such that $\sum_{i=1}^{n-1} \lambda_{i} \leqslant p-n$ and $\sum_{i=1}^{n-1} \mu_{i} \leqslant q-n$, there exist complex numbers $\left\{v_{3}, \ldots, v_{n}\right\}$ such that the induced module $M\left(N, v_{3}, \ldots, v_{n}\right)$ is a simple admissible generic $\mathfrak{s l}_{n}$-module in the principal orbit. Moreover, these modules exhaust all simple weight admissible generic $\mathfrak{s l}_{n}$-modules in the principal orbit induced from $\mathfrak{s l}_{2}$.

Proof. Note that $N$ belongs to the nilpotent orbit and, hence, strongly generic. Set $v_{1}=u_{21}, v_{2}=u_{22}$ and choose $v_{3}, \ldots, v_{n}$ such that $v_{i}-v_{i+1}-1=\lambda_{i}-\frac{p}{q}\left(\mu_{i}+1\right)$ for any $i=1, \ldots, n-1$. The statements follow from Lemma 6.2.

Remark 6.6. Theorem 6.5 is a ratification of Corollary 4.3 for generic modules in the principal orbit. In order to complete a classification of all simple admissible $\mathfrak{s l}_{2}{ }^{-}$ induced modules one needs to consider non-principal orbits and singular modules. In the case of $\mathfrak{s l}_{3}$ such modules were constructed in Theorem 5.6 and Theorem 5.8.

6.3. Admissible $\mathfrak{s l}_{3}$-induced modules. Any simple admissible $\mathfrak{s l}_{3}$-module in the principal nilpotent orbit admits a tableaux realization as a subquotient of some Gelfand-Tsetlin module $V(T(u))$ where $u=\left(u_{31}, u_{32}, u_{33}, u_{21}, u_{22}, u_{11}\right)$ and for $i=$ $1,2, u_{3 i}-u_{3, i+1}-1=\lambda_{i}-\frac{p}{q}\left(\mu_{i}+1\right)$ for some $\lambda_{i}, \mu_{i} \in \mathbb{Z}_{\geqslant 0}$ satisfying $\lambda_{1}+\lambda_{2} \leqslant p-3$ 
and $\mu_{1}+\mu_{2} \leqslant q-3$ with $p, q \in \mathbb{N},(p, q)=1, p, q \geqslant 3$. We say in this case that $V(T(u))$ (and its simple quotient which contains $T(u))$ is associated with the weight $\left(\lambda_{1}-\frac{p}{q}\left(\mu_{1}+1\right), \lambda_{2}-\frac{p}{q}\left(\mu_{2}+1\right)\right)$.

Theorem 6.7. Let $N$ to be a simple admissible $\mathfrak{s l}_{3}$-module in the principal nilpotent orbit associated with the weight $\lambda^{\prime}=\left(\lambda_{1}-\frac{p}{q}\left(\mu_{1}+1\right), \lambda_{2}-\frac{p}{q}\left(\mu_{2}+1\right)\right)$ such that $\lambda_{1}+\lambda_{2} \leqslant p-n$ and $\mu_{1}+\mu_{2} \leqslant q-n$ and $p, q \geqslant n$. Let $\left\{\lambda_{i}, \mu_{i}\right\}_{i=3, \ldots, n-1} \subseteq \mathbb{Z}_{\geqslant 0}$ such that $\sum_{i=1}^{n-1} \lambda_{i} \leqslant p-n$ and $\sum_{i=1}^{n-1} \mu_{i} \leqslant q-n$. Set $v_{1}=u_{31}, v_{2}=u_{32}, v_{3}=u_{33}$ and choose complex numbers $\left\{v_{4}, \ldots, v_{n}\right\}$ such that $v_{i}-v_{i+1}-1=\lambda_{i}-\frac{p}{q}\left(\mu_{i}+1\right)$ for any $i=1, \ldots, n-1$. Then the induced module $M\left(N, v_{4}, \ldots, v_{n}\right)$ is an admissible $\mathfrak{s l}_{n^{-}}$ module in the principal orbit. Moreover, simple modules $M\left(N, v_{4}, \ldots, v_{n}\right)$ exhaust all simple admissible $\mathfrak{s l}_{3}$-induced modules in the principal nilpotent orbit.

Proof. The statement is clear from the construction.

Theorem 6.7 together with Lemma 6.3 and Theorem 5.8 give a complete classification of all simple weight admissible $\mathfrak{s l}_{3}$-induced modules $M\left(N, v_{4}, \ldots, v_{n}\right)$ in the principal nilpotent orbit.

Remark 6.8. Theorem 6.7 describes both generic and singular $\mathfrak{s l}_{3}$-induced modules $M\left(N, v_{4}, \ldots, v_{n}\right)$. All these modules have infinite weight multiplicities. Generic modules were described already in Corollary 4.3.

\section{REFERENCES}

[Ad16] Dražen Adamović, A realization of certain modules for the $N=4$ superconformal algebra and the affine Lie algebra $A_{1}^{(1)}$,Transformation Groups, Vol. 21, No. 2 2016, pp. 299-327.

[AM95] Dražen Adamović and Antun Milas. Vertex operator algebras associated to modular invariant representations for $A_{1}^{(1)}$. Math. Res. Lett., 2(5):563-575, 1995.

[Ara14] Tomoyuki Arakawa. Two-sided BGG resolution of admissible representations. Represent. Theory, 18(3):183-222, 2014.

[Ara15a] T. Arakawa. Associated varieties of modules over Kac-Moody algebras and $C_{2}$ cofiniteness of W-algebras. Int. Math. Res. Not., 2015:11605-11666, 2015.

[Ara15b] T. Arakawa. Rationality of W-algebras: principal nilpotent cases. Ann. Math., 182(2):565-694, 2015.

[Ara16] T. Arakawa. Rationality of admissible affine vertex algebras in the category $\mathcal{O}$. Duke Math. J., 165(1):67-93, 2016.

[CR12] T. Creutzig, D. Ridout. Modular data and Verlinde formulae for fractional level WZW models I. Nuclear Phys. B, 865(1):83-114, 2012.

[CR13] T. Creutzig, D. Ridout. Modular data and Verlinde formulae for fractional level WZW models II. Nuclear Phys. B, 875(2):423-458, 2013.

[Deo80] V. Deodhar, On a construction of representations and a problem of Enright, Invent. Math. 57 (1980), 101-118.

[DMP00] I. Dimitrov, O. Mathieu, I. Penkov, On the structure of weight modules, Trans. Amer. Math. Soc. 352 (2000), 2857-2869.

[DFO89] Y. Drozd, S. Ovsienko, V. Futorny, Irreducible weighted $\mathfrak{s l}(3)$-modules, Funksionalnyi Analiz i Ego Prilozheniya 23 (1989), 57-58.

[DFO92] Y. Drozd, V. Futorny, S. Ovsienko, Gelfand-Tsetlin modules over Lie algebra sl(3), Contemp. Math. 131 (1992) 23-29.

[DFO94] Y. Drozd, V. Futorny, and S. Ovsienko, Harish-Chandra subalgebras and Gelfand-Zetlin modules, Finite-dimensional algebras and related topics (Ottawa, ON, 1992) NATO Adv. Sci. Inst. Ser. C Math. Phys. Sci., vol. 424, Kluwer Acad. Publ., Dordrecht, 1994, pp. 79-93.

[Duf77] M. Duflo. Sur la classification des idéaux primitifs dans l'algèbre enveloppante d'une algèbre de Lie semi-simple. Ann. of Math. (2), 105(1):107-120, 1977. 
[FST] B. L. Feigin, A. M. Semikhatov and I. Yu. Tipunin, Equivalence between chain categories of representations of affine $s l(2)$ and $N=2$ superconformal algebras. J. Math. Phys. 39 (1998), 3865-390.

[Fe90] S. Fernando, Lie algebra modules with finite dimensional weight spaces I, Trans. Amer. Math. Soc. 322 (1990), 757-781.

[FO10] V. Futorny, S. Ovsienko, Galois orders in skew monoid rings, J. Algebra 324 (2010), 598-630

[FO14] V. Futorny, S. Ovsienko, Fibers of characters in Gelfand-Tsetlin categories, Trans. Amer. Math. Soc. 366 (2014), 4173-4208.

[FBZ04] E. Frenkel, D. Ben-Zvi. Vertex algebras and algebraic curves, volume 88 of Mathematical Surveys and Monographs. American Mathematical Society, Providence, RI, second edition, 2004

[FKW92] E. Frenkel, V. Kac, M. Wakimoto. Characters and fusion rules for $W$-algebras via quantized Drinfel'd-Sokolov reduction. Comm. Math. Phys., 147(2):295-328, 1992

[FGR15] V. Futorny, D. Grantcharov, L.E. Ramirez. Irreducible generic Gelfand-Tsetlin modules of $\mathfrak{g l}(n)$, SIGMA 11 (2015) 018, 13 pp.

[FGR16] V. Futorny, D. Grantcharov, L. E. Ramirez, Singular Gelfand-Tsetlin modules for $\mathfrak{g l}(n)$. Advances in Mathematics, Vol 290, 2016, 453-482.

[FGR] V. Futorny, D. Grantcharov, L.E. Ramirez. Classification of irreducible Gelfand-Tsetlin modules for $\mathfrak{s l}(3)$. In progress.

[GT50] I. Gelfand, M. Tsetlin. Finite-dimensional representations of groups of orthogonal matrices, Doklady Akad. Nauk SSSR (N.S.) 71 (1950), 1017-1020 (Russian).

[GR04] M. Graev, Infinite-dimensional representations of the Lie algebra $g l(n, \mathbb{C})$ related to complex analogs of the Gelfand-Tsetlin patterns and general hupergeometric functions on the Lie group $G L(n, \mathbb{C})$, Acta Appl. Mathematicae 81 (2004), 93-120.

[Jan77] J. C. Jantzen. Kontravariante Formen auf induzierten Darstellungen halbeinfacher LieAlgebren. Math. Ann., 226(1):53-65, 1977.

[Jos85] Anthony Joseph. On the associated variety of a primitive ideal. J. Algebra, 93(2):509$523,1985$.

[Jos79] A. Joseph. Dixmier's problem for Verma and principal series submodules. J. London Math. Soc. (2), 20(2):193-204, 1979.

[Kac98] V. Kac. Vertex algebras for beginners, volume 10 of University Lecture Series. American Mathematical Society, Providence, RI, second edition, 1998.

[KW89] V. G. Kac and M. Wakimoto. Classification of modular invariant representations of affine algebras. In Infinite-dimensional Lie algebras and groups (Luminy-Marseille, 1988), volume 7 of Adv. Ser. Math. Phys., pages 138-177. World Sci. Publ., Teaneck, NJ, 1989.

[KW08] V. Kac, M. Wakimoto. On rationality of $W$-algebras. Transform. Groups, 13(3-4):671713,2008

[Kho05] O. Khomenko. Some applications of Gelfand-Zetlin modules, Representations of algebras and related topics, Fields Inst. Commun., vol. 45, Amer. Math. Soc., Providence, RI, 2005, pp. 205-213.

[KW06] B. Kostant, N. Wallach, Gelfand-Zeitlin theory from the perspective of classical mechanics I, In Studies in Lie Theory Dedicated to A. Joseph on his Sixtieth Birthday, Progress in Mathematics, 243 (2006), 319-364.

[KW6] B. Kostant, N. Wallach, Gelfand-Zeitlin theory from the perspective of classical mechanics II. In The Unity of Mathematics In Honor of the Ninetieth Birthday of I. M. Gelfand, Progress in Mathematics, 244 (2006), 387-420.

[Mat00] O. Mathieu. Classification of irreducible weight modules. Ann. Inst. Fourier (Grenoble), 50(2):537-592, 2000.

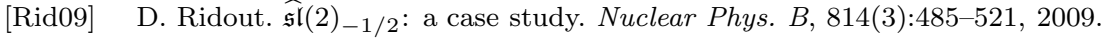

[Rid10] D. Ridout. $\widehat{\mathfrak{s l}}(2)_{-1 / 2}$ and the triplet model. Nuclear Phys. B, 835(3):314-342, 2010.

[Wan99] W. Wang. Dimension of a minimal nilpotent orbit. Proc. Amer. Math. Soc., 127(3):935936, 1999.

[Zhu96] Y. Zhu. Modular invariance of characters of vertex operator algebras. J. Amer. Math. Soc., 9(1):237-302, 1996.

[Zh74] D. Zhelobenko, Compact Lie groups and their representations, Transl. Math. Monographs, AMS, 40 (1974) 
Research Institute for Mathematical Sciences, Kyoto University, Kyoto 606-8502 JAPAN

E-mail address: arakawa@kurims.kyoto-u.ac.jp

Instituto de Matemática e Estatística, Universidade de São Paulo, São Paulo-SP, BRAZIL

E-mail address: futorny@ime.usp.br

Universidade Federal do ABC, SAnto André-SP, Brazil

E-mail address: luis.enrique@ufabc.edu.br 